\title{
Neutrophils are mediators of metastatic prostate cancer progression in bone
}

\author{
Diane L. Costanzo-Garvey ${ }^{1}$. Tyler Keeley ${ }^{1} \cdot$ Adam J. Case $^{2} \cdot$ Gabrielle F. Watson $^{2} \cdot$ Massar Alsamraae $^{1}$. \\ Yangsheng $\mathrm{Yu}^{1} \cdot \mathrm{Kaihong} \mathrm{Su}^{1,5}$. Cortney E. Heim ${ }^{1} \cdot$ Tammy Kielian $^{1} \cdot$ Colm Morrissey $^{3} \cdot$ Jeremy S. Frieling $^{4}$. \\ Leah M. Cook ${ }^{1}$ (i)
}

Received: 24 July 2019 / Accepted: 17 February 2020 / Published online: 29 February 2020

(c) The Author(s) 2020

\begin{abstract}
Bone metastatic prostate cancer (BM-PCa) significantly reduces overall patient survival and is currently incurable. Current standard immunotherapy showed promising results for PCa patients with metastatic, but less advanced, disease (i.e., fewer than 20 bone lesions) suggesting that PCa growth in bone contributes to response to immunotherapy. We found that: (1) PCa stimulates recruitment of neutrophils, the most abundant immune cell in bone, and (2) that neutrophils heavily infiltrate regions of prostate tumor in bone of BM-PCa patients. Based on these findings, we examined the impact of direct neutrophilprostate cancer interactions on prostate cancer growth. Bone marrow neutrophils directly induced apoptosis of PCa in vitro and in vivo, such that neutrophil depletion in bone metastasis models enhanced BM-PCa growth. Neutrophil-mediated PCa killing was found to be mediated by suppression of STAT5, a transcription factor shown to promote PCa progression. However, as the tumor progressed in bone over time, neutrophils from late-stage bone tumors failed to elicit cytotoxic effector responses to PCa. These findings are the first to demonstrate that bone-resident neutrophils inhibit PCa and that BM-PCa are able to progress via evasion of neutrophil-mediated killing. Enhancing neutrophil cytotoxicity in bone may present a novel therapeutic option for bone metastatic prostate cancer.
\end{abstract}

Keywords Neutrophils $\cdot$ Bone $\cdot$ Metastasis $\cdot$ Prostate $\cdot$ Cancer $\cdot$ STAT5

Abbreviations
$\begin{array}{ll}\text { BM-PCa } & \text { Bone metastatic prostate cancer } \\ \text { CM } & \text { Conditioned media } \\ \text { DHR 1,2,3 } & \text { Dihydrorhodamine 1,2,3 }\end{array}$

Electronic supplementary material The online version of this article (https://doi.org/10.1007/s00262-020-02527-6) contains supplementary material, which is available to authorized users.

Leah M. Cook

leah.cook@unmc.edu

1 Department of Pathology and Microbiology, University of Nebraska Medical Center, 985900 Nebraska Med Center, Omaha, NE 68192, USA

2 Department of Cellular and Integrative Physiology, University of Nebraska Medical Center, Omaha, NE, USA

3 Department of Urology, University of Washington, Seattle, WA, USA

4 Tumor Biology Department, H. Lee Moffitt Cancer Center and Research Institute, Tampa, FL, USA

5 Department of Medical Education, California University of Science and Medicine, San Bernadino, CA, USA

$\begin{array}{ll}\text { HSC } & \text { Hematopoietic stem cell } \\ \text { IL-8 } & \text { Interleukin } 8 \\ \text { LPS } & \text { Lipopolysaccharide } \\ \text { MDSC } & \text { Myeloid-derived suppressor cell } \\ \text { MPO } & \text { Myeloperoxidase } \\ \text { MSC } & \text { Mesenchymal stem cell } \\ \text { NE } & \text { Neutrophil elastase } \\ \text { NET } & \text { Neutrophil extracellular trap } \\ \text { PMA } & \text { Phorbol 12-myristate 13-acetate } \\ \text { ROS } & \text { Reactive oxygen species } \\ \text { TGF } \beta & \text { Transforming growth factor beta } \\ \text { T } \beta \text { RI } & \text { Transforming growth factor beta receptor I }\end{array}$

\section{Introduction}

Approximately $90 \%$ of men with advanced prostate cancer $(\mathrm{PCa})^{1}$ present with bone metastases. Bone is the preferential site for PCa metastasis and is associated with increased risk of fractures, spinal cord compression, and severe pain [1]. Although current standard-of-care bone- and 
osteoclast-targeted therapies, such as radium-223 and denosumab, delay tumor growth and cancer-induced bone disease, respectively, these have been unsuccessful in eliminating and preventing bone metastatic $\mathrm{PCa}(\mathrm{BM}-\mathrm{PCa})$ growth in bone [2]. Patients with localized disease have a significantly better outcome than BM-PCa patients, with 5-year survival being reduced from nearly $100 \%$ for localized disease to less than $3 \%$ of bone metastatic disease [1]. To date, bone metastatic $\mathrm{PCa}(\mathrm{BM}-\mathrm{PCa})$ remains incurable.

PCa cells thrive in the bone microenvironment by hijacking the coupled process of bone remodeling characterized by osteoclast-mediated bone degradation and osteoblastmediated bone formation. Heightened bone turnover results in the release of bone sequestered factors such as transforming growth factor beta (TGF $\beta$ ) [3] that promotes increased cancer cell survival and growth. While many studies have focused on the interplay between PCa cells, osteoblasts, and osteoclasts, it is increasingly evident that a large array of cell types in the bone microenvironment including mesenchymal stem cells (MSCs), hematopoietic stem cells (HSCs) and immune cells can contribute to the progression of these bone metastases [4].

Previously, we observed that $\mathrm{PCa}$ can regulate bone formation by recruiting MSCs, which in turn differentiate into bone-forming osteoblasts [5]. We also examined the differential effect of PCa cells on bone marrow MSC gene expression and observed a significant induction of interleukin (IL-8) in MSCs in response to PCa conditioned media. IL-8 is known to contribute to osteoclast formation but also is a potent chemoattractant for neutrophils [6]. Neutrophils and neutrophil precursors are the most abundant immune cell in bone, $\sim 60 \%$ and $\sim 40 \%$ of the human and mouse bone marrow compartment, respectively $[7,8]$. Neutrophils are generated in the bone marrow at rates of $10^{11}$ cells daily and are regularly released into circulation [9]. Bodily infection or tissue damage results in a systemic gradient of chemokines being released, including IL-8 and CXCL5, which causes rapid neutrophil expansion and mobilization from the bone marrow or blood circulation into tissues followed by neutrophil secretion of a number of effector molecules, including bactericidal enzyme-containing granules, reactive oxygen species (ROS), and neutrophil extracellular traps (NETs), mesh-like scaffolds of decondensed DNA and granule enzymes [10]. In the past decade, emerging evidence has demonstrated a role for neutrophils in cancer progression, albeit conflicting, with data indicating both anti- and protumor properties [11]. Recently, the existence of a protumoral/“N2" and an anti-tumoral/"N1," phenotype in the tumor microenvironment, has been described with emergence and dominance of the $\mathrm{N} 2$ subtype being regulated by TGF $\beta$ [12]. Surprisingly, given their abundance in the bone marrow, the role of neutrophils in the progression of bone metastatic prostate cancer has not been examined thus far.
In the current study, we established the presence of neutrophils in human BM-PCa biopsies and demonstrated that PCa cells stimulate neutrophil migration, oxidative burst, and NET formation, properties associated with neutrophil activation. Reciprocally, direct co-culture assays and live cell imaging revealed that neutrophils induce PCa apoptosis. In support of this, depletion of neutrophils in vivo accelerated the growth of BM-PCa in two independent mouse models of bone metastatic prostate cancer. This phenomenon appears to be regulated by neutrophil inhibition of STAT5 signaling in PCa. Additionally, we observed that the cytotoxic neutrophil phenotype was diminished as BM-PCa progresses in bone. Collectively, these findings are the first to demonstrate an anti-tumor role for neutrophils in the prostate tumor-bone microenvironment and demonstrate that BM-PCa progresses in bone via evasion of neutrophil-mediated PCa death.

\section{Materials and methods}

\section{Tissues and cell lines}

Luciferase-expressing cell lines were generated by the Cook Laboratory using a lentiviral luciferase reporter (Qiagen), according to protocol. To collect conditioned media (CM), cell lines were rinsed with Phosphate Buffered Saline (PBS) to remove serum, complete medium was replaced with serum-free medium and cells were incubated overnight. $\mathrm{CM}$ was collected $18-20 \mathrm{~h}$ later by brief centrifugation to remove cellular debris and stored at $4{ }^{\circ} \mathrm{C}$ until usage. To minimize media-based changes, all $\mathrm{CM}$ was collected in RPMI. Total protein content of CM was measured using BCA assay (ThermoFisher) to ensure equal protein concentrations for treating neutrophils. Fresh media was collected every 2 weeks for experimental use.

\section{Neutrophil isolation from bone marrow}

Human bone marrow was processed using a modified Ficoll density centrifugation protocol. Briefly, bone marrow was washed in neutrophil isolation buffer at 7:1 ratio to bone marrow. This suspension was filtered with a $70-\mu \mathrm{M}$ filter to remove bone fragments. Diluted bone marrow was slowly pipetted onto $15 \mathrm{~mL}$ of Ficoll-Paque in a $50-\mathrm{mL}$ conical tube and centrifuged at $445 \times \mathrm{g}$ for $35 \mathrm{~min}$ at room temperature with no centrifuge brake. The granulocyte layer (the lower layer) was carefully removed and washed twice with buffer, and cells were counted for subsequent experiments. For mouse neutrophil isolation, mouse tibia and femurs were removed from male C57BL/6 mice. Bones were cleared of tissue and muscle, and the epiphysis was removed and discarded. A hole was made in the bottom of a $0.65 \mathrm{~mL}$ tube, and one bone was placed individually per tube. This tube 
was placed into a $1.5-\mathrm{mL}$ tube for bone marrow collection, where it was then centrifuged at high speed for $<5 \mathrm{~s}$. The bone marrow was re-suspended in $1 \mathrm{~mL}$ of neutrophil isolation buffer and filtered using a $70-\mu \mathrm{M}$ filter. Bones from each mouse were pooled and counted, and the EasySep Mouse Neutrophil Enrichment (Stem Cell Technologies) protocol was followed, as per manufacturers' instructions. Neutrophil purity was validated using flow cytometry for specific mark-

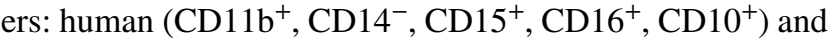
mouse $\left(\mathrm{CD} 45^{+}, \mathrm{CD} 11 \mathrm{~b}^{+}, \mathrm{Ly}_{6 \mathrm{G}}{ }^{\mathrm{hi}}\right)$. All neutrophil described functional assays utilized mouse bone marrow neutrophils, and major findings were validated in human neutrophils.

\section{Cell culture media, reagents, and buffers}

Neutrophil isolation buffer consists of 1X PBS, 2\% FBS and 2 mM EDTA. RPMI complete media consists of RPMI (Hyclone), $10 \%$ FBS (Peak Serum), and 1\% penicillin/ streptomycin. DMEM complete media consists of DMEM (Hyclone), 10\% FBS (Peak Serum), and 1\% penicillin/streptomycin. Luciferase-expressing C42B and PAIII were supplemented with $10 \mu \mathrm{g}$ of puromycin, and LNCaP with $5 \mu \mathrm{g}$ puromycin (Gibco). For oxidative burst assays, cells were treated with either serum-free CM, lipopolysaccharide (LPS; $5 \mu \mathrm{g} / \mathrm{ml}$, Sigma), or phorbol 12-myristate 13-acetate (PMA; $5 \mathrm{nM}$, Sigma) in serum-containing (10\%) RPMI. For T $\beta$ RI assays, primary neutrophils were incubated in CM supplemented with RepSox (5 nM; Selleckchem).

\section{Neutrophil migration assay}

Permeable 24-well transwell migration chambers were used with a pore size of $5 \mu \mathrm{m}$ (Costar; Ref \# 3422). Primary neutrophils were isolated from mouse hind limbs and $1 \times 10^{5}$ were seeded in $250 \mu \mathrm{L}$ serum-free media in the inserts and $650 \mu \mathrm{L}$ of conditioned media was added to the bottom of the respective wells, and then inserts were carefully lowered into the wells ensuring that no bubbles formed between the membrane and top of the conditioned media. Neutrophils were incubated for $1 \mathrm{~h}$ at $37^{\circ} \mathrm{C}$, then inserts were rinsed in $1 \mathrm{x}$ PBS and fixed overnight in methanol at $-20{ }^{\circ} \mathrm{C}$. Fixed inserts were stained with hematoxylin for quantitation of number of migrating neutrophils per insert. Because $~ 90 \%$ of the neutrophils completely migrated through the inserts, neutrophils in the lower chamber media were counted using Trypan Blue Exclusion assay. For investigating the importance of IL8/CXCL1 in PCa-mediated neutrophil migration, bone-derived mouse neutrophils were pre-treated with an antibody to mouse CXCR2 (50 nM; Cayman Chemicals) for $30 \mathrm{~min}$ and CellTracker Green (Invitrogen) prior to addition to inserts and allowed to migrate for $1 \mathrm{~h}$ towards specific PCa CM. After inserts were removed, the wells were imaged using an EVOS FL Auto microscope (Invitrogen;
AMAFD1000) at 10x to quantify neutrophils that completely penetrated the membrane.

\section{Immunofluorescence}

Paraffin-embedded patient bone specimens and mouse tibia bone sections were dewaxed and hydrated through an alcohol gradient. Antigen retrieval for human and mouse specimens was performed using Tris-EDTA buffer ( $\mathrm{pH} 9)$ in a pressure cooker for $6 \mathrm{~min}$. Tissues were then blocked in $10 \%$ serum in $1 \mathrm{X}$ Tris-buffered saline (TBS) for $1 \mathrm{~h}$ prior to overnight incubation with primary antibodies (Cytokeratin (dilution 1:500), Sigma C2562; phospho-histone H3 (dilution 1:200), Cell Signaling 9701L; Neutrophil Elastase (dilution 1:200), Abcam ab68672; Myeloperoxidase (dilution $7.5 \mu \mathrm{g}$ / ml), R\&D AF3667). Following washes, species-specific secondary AlexaFluor antibodies were incubated 1:1000 on the tissues for $1 \mathrm{~h}$ at room temperature (A11029, A11036, A21206, and A11057). Fluorescent images were taken on a Zeiss Axio Imager.Z2 at 20x and quantified using ImageJ. Phospho-histone H3 was quantified as the number of positive cells compared to the total number of cells per image as determined by DAPI staining. STAT5 was quantified as the amount of positive STAT5 signal per total area of tumor tissue. A threshold for STAT5 signal was set for all images analyzed, and the percent of positive pixels was obtained for each image. Using cytokeratin, the total tumor area in pixels was also obtained. By multiplying the percentage of STAT5 and cytokeratin-positive pixels with the image area, we were able to obtain tumor area and STAT5 positive area. Dividing the calculated STAT5 area by the cytokeratin area yielded the percent of STAT5 per tumor area in each image.

\section{Flow cytometry}

Isolated neutrophils were transferred to FACS buffer ( $2 \%$ FBS in $1 X$ PBS) at $1 \times 10^{6}$ cells in $200 \mu \mathrm{L}$. For tumor studies, bone marrow was flushed from tumor-bearing and saline-injected tibiae using a syringe and excess cells were further flushed out of the marrow with EasySep buffer. Cells were treated for 2-3 min in 1X Red Blood Cell Lysis Buffer (BioLegend) at room temperature, washed in 1X PBS, and counted for antibody staining. For staining, cells were incubated on ice with $1 \mu \mathrm{L}$ per $10^{6}$ cells in mouse or human TruStain Fc block (Biolegend) for $10 \mathrm{~min}$. Fluorophoreconjugated antibodies were added at a maximum of $1 \mu \mathrm{L}$ per $10^{6}$ cells (Human-APC/Cy7-CD11b, PE/Cy7-CD14, FITC-CD15, PerCp-Cy5.5-CD10; Mouse- APC-CD45, FITC-CD11b, PE-Ly6G, PerCp-Cy5.5-Ly6C). Cell viability dye, Live/Dead (Invitrogen), was added at a concentration of $0.2 \mu \mathrm{L}$ per $10^{6}$ cells. Stained cells were incubated with antibody on ice in the dark for $20 \mathrm{~min}$ and rinsed with $1 \mathrm{X}$ PBS. Cells were fixed by incubation in $1 \%$ formaldehyde 
in 1XPBS for $30 \mathrm{~min}$ in the dark and rinsed with 1X PBS. Prior to analysis, cells were reconstituted in FACS buffer. For all analyses, single cells were gated and marker expression analyzed in myeloid cells.

\section{Oxidative burst assay}

Primary mouse neutrophils were isolated from whole bone marrow (EasySep). Neutrophils were incubated under sterile conditions in serum-free conditioned media from LNCaP, C42B, PC3, BPH, and RWPE-1 or appropriate serum-free base medium for an hour at $37^{\circ} \mathrm{C}$ in flow cytometry tubes. For a positive control of neutrophil activation and oxidative burst, neutrophils were treated with lipopolysaccharide (LPS; $5 \mu \mathrm{g} / \mathrm{mL}$ ) for $30 \mathrm{~min}$ in serum-containing RPMI. After incubation in prostate CM, dihydrorhodamine 1,2,3 (DHR123; $25 \mu \mathrm{M}$ ) was added to neutrophils in each CM condition, allowed to incubate for 30 additional minutes at $37^{\circ} \mathrm{C}$ and cell fluorescence was analyzed via flow cytometry. Immediately prior to flow analysis, DAPI was added to each condition to measure cell viability. For a longitudinal analysis of oxidative burst, primary neutrophils were placed in a 96-well plate in prostate CM and DHR123 immediately added to each well. Green fluorescence/oxidized DHR was measured over time using an Incucyte S3 Live-Cell Analysis System Imager.

\section{Sytox green/NET formation assay}

For analysis of NET formation, primary mouse neutrophils were incubated for $2 \mathrm{~h}$ in prostate $\mathrm{CM}$ (LNCaP, C42B, PC3, BPH, and RWPE-1) or complete RPMI supplemented with PMA (100 nM) as a positive control. Sytox Green dye (500 nM; Sigma) was added to each condition, and after 30 min, images were taken of each well by an EVOS FL Auto microscope. The number of green fluorescent secreted DNA traps/NETs was measured as a percentage of total cells to distinguish between dying cells that absorbed the Sytox Green dye. CM-treated neutrophils were compared to neutrophils incubated in serum-free media RPMI.

\section{Real-time qPCR}

For analysis of neutrophils gene expression, RNA was isolated from neutrophils which had been incubated for $3 \mathrm{~h}$ at $37{ }^{\circ} \mathrm{C}$, in PCa CM supplemented with $2 \%$ FBS. RNA was extracted using Trizol. RNA $(1 \mu \mathrm{g})$ was used to synthesize cDNA using qSCRIPT Super mix (Quantabio) and PCR performed using Perfecta SYBR Green FastMix (Quantabio). PCR was run using Bio-Rad CFX Real-Time System. PCR conditions were as follows for all primer sequences (Supplemental Table 1): Step 1: $95^{\circ} 30 \mathrm{~s}$; Step 2: $95^{\circ} 5 \mathrm{~s}, 57^{\circ} 15 \mathrm{~s}$, $72^{\circ} 10 \mathrm{~s}, 95^{\circ} 10 \mathrm{~s}(\times 39$ cycles $)$; Step 3 : Melt curve $65^{\circ} 95^{\circ}$ increments of $0.5^{\circ}$ for $5 \mathrm{~s}$.

\section{PCa co-cultures with neutrophils}

Luciferase-expressing $\mathrm{LNCaP}$ or $\mathrm{C} 42 \mathrm{~B}$ cells were plated at 40,000 cells/well in a 24 -well plate, in triplicate per condition. Twenty-four hours later, neutrophils were isolated, re-suspended in complete medium, and plated in direct contact with cancer cells at a ratio of 10:1 (neutrophils/cancer). After incubation overnight, neutrophils were removed, and cancer cell viability was measured with Trypan Blue Exclusion assay, using a hemacytometer.

\section{Incucyte S3 live-cell analysis and NanoLive}

$\mathrm{C} 42 \mathrm{~B}$ and $\mathrm{LNCaP}$ co-cultures were performed using the above conditions, with modifications in cell density. C42B and LNCaP cells were plated at 9000 cells/well in a 96 well plate in triplicate, per condition. Mouse neutrophils were isolated the following day and plated at a ratio of 10:1 (neutrophils/cancer). The 96-well plate was placed into an Incucyte S3 Live-Cell Analysis System S3 Live-Cell Imager after neutrophils were added. Live-cell images were taken every $10 \mathrm{~min}$ and data quantified over a 24 -h period. Three replicates of each cell line and condition were averaged, and data were analyzed using the Incucyte S3 Live-Cell Analysis System S3 analysis software (Essen biosciences). For imaging using NanoLive, C42B cells were plated in 6-well plates, and $24 \mathrm{~h}$ later, mouse bone marrow-derived neutrophils were added at a 10:1 neutrophil/cancer ratio and imaged for $1 \mathrm{~h}$, as described.

\section{Real-time Glo MT cell viability assay}

Neutrophils were incubated in triplicate at 100,000 per well in PCa CM with 2x Real-Time Glo reagent (Promega). MT Cell Viability Substrate and NanoLuc ${ }^{\circledR}$ Enzyme were added in equal volumes to culture media to create the $2 \mathrm{x}$ Real-time Glo reagent. This media was added directly to the cells at time zero, and luminescence was read at the indicated time points using a luminometer.

\section{In vivo mouse models}

Luciferase-expressing C42B, PAIII, and LNCaP cells were grown to confluence in complete media (base media, $10 \%$ fetal bovine serum, $1 \%$ penicillin/streptomycin). Cells were trypsinized and washed three times with $1 \mathrm{X}$ PBS and filtered using a $70-\mu \mathrm{M}$ nylon filter. Cells were counted and reconstituted for injection of appropriate cell numbers per $20 \mu \mathrm{L}$ volume per mouse $(500,000$ cells-LNCaP and C42B; 50,000-PAIII). Fox Chase SCID 
Beige male mice (Charles River) were anesthetized with isoflurane, and the right tibia was injected with $20 \mu \mathrm{L}$ of cells. An equal volume of PBS was injected into the contralateral limb as a control for the intratibial injection. For imaging of tumor burden via bioluminescence, mice were given $10 \mu \mathrm{L} /$ gram of D-luciferin $(15 \mathrm{mg} / \mathrm{mL})$ (Gold Bio) IP imaged using the IVIS Spectrum imager (PerkinElmer) on each day of antibody treatment. Luciferase signal was quantified 15 min after injection, using the Living Image Software per manufacturer's instructions. Using bioluminescence intensity, mice were randomized into 2 groups to receive either ( $n=5 /$ group): a rat $\operatorname{IgG} 2 \mathrm{~A}$ isotype control antibody or Anti-Ly6G clone 1A8 (BioXcell) to ensure there were no differences in tumor burden at the start of neutrophil depletion. For LNCaP and C42B studies, mice received an intraperitoneal (IP) dose of $400 \mu \mathrm{g} 1 \mathrm{~A} 8$ on day 3 post-inoculation, and subsequent doses $200 \mu \mathrm{g}$ twice per week to maintain low neutrophil numbers for the remainder of the study (for 2 weeks), based on previous experiments [13]. Depletion efficiency was determined by flow cytometry of bone marrow and spleen. For late-stage tumor depletion, C42B cells were intratibially injected in SCID Beige mice ( $n=10 /$ group) and after approximately 2 weeks were treated with isotype control or $1 \mathrm{~A} 8$ antibody for the remainder of the study. For PAIII, mice received an initial dose of $100 \mu \mathrm{g}$ antibody beginning on day 1 post-tumor inoculation and every other day for the remainder of the experiment. IVIS imaging was used for longitudinal measurement of tumor burden. To examine tumor-associated neutrophils throughout prostate cancer growth in bone, male SCID Beige mice $(n=15)$ were intratibially injected with 500,000 C42B cells in each limb and neutrophils were isolated from each tibia at 3 different time points after injection (week 1, week 2, and week 4). For control tumor-naïve neutrophil collections, additional mice $(n=5)$ were injected with saline. Mice were pooled per group and functional analyses performed: $\mathrm{PCa}$ co-culture assay, $\mathrm{T}$ cell suppression assay and cell viability.

\section{T cell proliferation assay}

$\mathrm{T}$ cell proliferation assays were performed with neutrophils recovered from the bones of tumor-bearing and control mice as previously described [14]. Naïve $\mathrm{CD} 4^{+} \mathrm{T}$ cells were isolated from the spleens of C57BL/6 mice by negative selection (Biolegend) and labeled with eFluor670 cell proliferation dye ( $5 \mu \mathrm{M}$; ThermoFisher), according to the manufacturer's instructions. T cells were plated at $1 \times 10^{4}$ per well in a round bottom 96-well plate in RPMI-1640 supplemented with 10\% FBS, $1 \%$ L-glutamine, $1 \%$ HEPES, $1 \%$ penicillin/streptomycin, $0.1 \% \beta$-mercaptoethanol and $100 \mathrm{ng} / \mathrm{ml}$ recombinant mouse IL-2 (BioLegend). Control or tumor-associated neutrophils isolated at week 1 and 4 post-intratibial injection were added at $1: 1$ or $5: 1$ ratio ( $\mathrm{T}$ cells:neutrophils) to $\mathrm{CD} 4^{+} \mathrm{T}$ cells subjected to polyclonal stimulation with anti-CD3/anti-CD28 Dynabeads (Gibco). TANs were isolated from tumor-bearing tibia, as described, and control neutrophils were isolated from saline-injected tibia.

\section{Trichrome staining}

Tibias isolated from mice were fixed in $10 \%$ neutral buffered formalin then stored in $70 \%$ ethanol. Tibia were decalcified in $14 \%$ EDTA buffer for 2 weeks at $4{ }^{\circ} \mathrm{C}$, changing the buffer every 2-3 days. Tibia were then embedded in paraffin and sectioned on a microtome to $5 \mu \mathrm{M}$ sections. Tissues were cleared of paraffin in 2 changes of xylene and hydrated through an alcohol gradient. Slides were then incubated in Bouin's fixative (Ricca, 1120-16) for $1 \mathrm{~h}$ at $55^{\circ} \mathrm{C}$. Slides were rinsed in running $\mathrm{dH} 2 \mathrm{O}$ until clear, then stained with hematoxylin (Ricca, 3530-32) for $5 \mathrm{~min}$. To blue the hematoxylin, slides were dipped in 1x TBS for $30 \mathrm{~s}$, then stained in Gomori's Trichrome solution (Volu-Sol, VXT-032) for $20 \mathrm{~min}$. Slides were then transferred to freshly made $0.5 \%$ acetic acid solution for $2 \mathrm{~min}$. The slides were rinsed in $\mathrm{dH} 2 \mathrm{O}$ until clear, then dehydrated, cleared in xylene, and mounted with Permount (Fisher, SP15-500). Images were taken at 4x, 10x, and 20x using an EVOS FL Auto microscope (Life Technologies, AMAFD1000). Areas of osteogenesis were quantified as percent of trabecular bone over total marrow area using ImageJ starting $500 \mu \mathrm{m}$ below the growth plate and continuing for $2 \mathrm{~mm}$.

\section{Proteome profiler human phospho-kinase array}

C42B and LNCaP cells were plated at 100,000 cells/well in a 6-well plate in triplicate, per condition. Mouse neutrophils were isolated the following day and plated at a ratio of 10:1 (neutrophils/cancer). $24 \mathrm{~h}$ later, the neutrophils were removed and protein was collected from lysed cancer cells for array analysis, performed per the manufacturer's instructions (R\&D Systems). Image $\mathbf{J}$ was used to measure array densitometry.

\section{Immunoblot analysis}

Whole-cell extracts were lysed in RIPA buffer and Halt protease and phosphatase inhibitor cocktail (ThermoFisher) was added as per manufacturers' instructions. Protein concentration was determined using BCA assay (ThermoFisher). Protein lysates were fractionated by SDS-PAGE and transferred to a nitrocellulose membrane using a transfer apparatus according to the manufacturer's protocols (Bio-Rad). After incubation with 5\% nonfat milk in $1 \mathrm{X}$ TBST for $1 \mathrm{~h}$, 
the membrane was washed once with TBST and incubated with appropriate antibodies (STAT5 (1:1000; Cell Signaling \#94205), $\beta$-actin (1:1000; Cell Signaling \#4970) or GAPDH (1:2000; Cell Signaling \#5174)) diluted in 1X TBST with 5\% milk. Phosphorylated STAT5a/b (1:1000; Cell Signaling \#4322) was diluted in $1 \mathrm{X}$ TBST with 5\% BSA. Membranes were incubated with rotation at $4{ }^{\circ} \mathrm{C}$ overnight. After incubation, primary antibody was removed and membranes were washed three times for $10 \mathrm{~min}$ each in $1 \mathrm{X}$ TBST and incubated with a 1:5000 dilution of horseradish peroxidaseconjugated anti-rabbit antibodies (Cell Signaling) for $1 \mathrm{~h}$ at room temperature. Blots were washed with $1 \mathrm{X}$ TBST three times and developed with the Clarity Western System (BioRad) according to the manufacturer's protocols. Blots were imaged on a digital CCD developer (Azure Biosystems).

\section{Generation of STAT5 knockdown cells}

STAT5A gene expression was reduced in C42B PCa cells using a STAT5A-targeted HuSH-29 shRNA lentiviral vector, which expresses red fluorescent protein (RFP). C42B cells were transfected using Lipofectamine, and RFP-positive cells were purified by FACS. As a control, C42B were transfected with a non-targeted scrambled sequence HuSH-29 shRNA vector. Three knockdown clones A, C and D were examined in direct co-cultures with mouse neutrophils in comparison to scrambled control C42B.

\section{Statistical analysis}

Statistical analyses ( $t$ test, ANOVA) were performed using GraphPad Prism (GraphPad Software, Inc). Error bars represent standard error from the mean (SEM).

\section{Results}

\section{Neutrophils co-localize with PCa cells in the tumor-bone microenvironment}

Previously, in examining the effect of bone metastatic $\mathrm{PCa}$ cells (C42B and PC3) on human MSC gene expression, we observed that IL- 8 was highly induced in MSCs in response to prostate cancer derived factors [15]. IL-8 is a potent neutrophil chemoattractant, and we reasoned that the interplay between prostate cancer cells and bone marrow MSCs could result in the recruitment of neutrophils within the bonePCa microenvironment. We examined whether this was the case in human samples of bone metastatic prostate cancer $(n=7)$. Immunofluorescence staining for the neutrophilspecific markers, neutrophil elastase (NE) and myeloperoxidase (MPO), revealed neutrophils proximal to prostate cancer cells (in Patient 1-5) (Fig. 1a, Supp. Figures 1A).
This contrasted from areas of "normal" bone marrow tissue in which the neutrophils appeared to be more evenly distributed (Supp. Figure 1A). Similarly, in a mouse model of bone metastatic PCa (C42B), we noted NE-positive neutrophils at the tumor-bone interface (Supp. Figure 1B, bottom). These findings suggest that neutrophils in bone may be localized to regions of metastatic PCa. However, to determine whether PCa directly influences neutrophil recruitment, primary mouse bone marrow neutrophils were allowed to migrate toward either serum-free medium (SFM; as a negative control), SFM supplemented with $2 \%$ FBS (positive control), or media from human $\mathrm{LNCaP}$ (non/poorly metastatic $\mathrm{PCa}$ ) cells or C42B (bone metastatic PCa cells derived from $\mathrm{LNCaP}$ ) in modified Boyden chamber assays. We observed that both LNCaP and C42B similarly enhanced neutrophil recruitment (Fig. 1b) independently of CXCL1/8, the mouse homologues of IL-8, demonstrated by blockade of neutrophil CXCR2 (Fig. 1c). These findings collectively suggest that neutrophils in bone are recruited via PCa-derived soluble factors.

\section{PCa induces neutrophil oxidative burst and NET formation}

Based on our evidence of PCa-mediated neutrophil recruitment, we next examined the impact of PCa on neutrophil function. Oxidative burst is a classical neutrophil cytotoxic response against pathogens. However, in the tumor microenvironment, oxidative burst has been shown to both inhibit tumor growth and to promote tumor growth indirectly via suppression of $\mathrm{T}$ cell activation [16]. To determine the impact of BM-PCa on neutrophil oxidative burst, primary bone marrow-derived mouse neutrophils were treated with PCa-derived CM from (1) poorly metastatic LNCaP or (2) bone metastatic $\mathrm{C} 42 \mathrm{~B}$, in comparison with non-malignant RWPE prostate epithelial cells. For negative and positive controls, neutrophils were treated with either SFM or serumcontaining medium supplemented with LPS (or PMA), an inducer of oxidative burst. Reactive oxygen species (ROS), as a measure of oxidative burst, was measured by dihydrorhodamine (DHR) 1,2,3, a dye that passively diffuses into cells and, upon oxidation, emits green fluorescence as a readout for ROS production [17]. Surprisingly, we observed that neutrophils exposed to bone metastatic C42B $\mathrm{CM}$ for $2 \mathrm{~h}$ produced more ROS than those treated with $\mathrm{LNCaP}(\sim$ sevenfold more $)$ and RWPE cell line media $(\sim$ sixfold more) and 2.5-fold more than the positive control, LPS (Fig. 2a, Supp. Figure 2A). However, longitudinal measurement of oxidative burst revealed that all of the prostate cancer cells and benign prostate hyperplasia (BPH-1) cells induced neutrophil oxidative burst, although at different time points. Specifically, LNCaP quickly activated neutrophils within the first 30 min of treatment, whereas PC3- and 
a

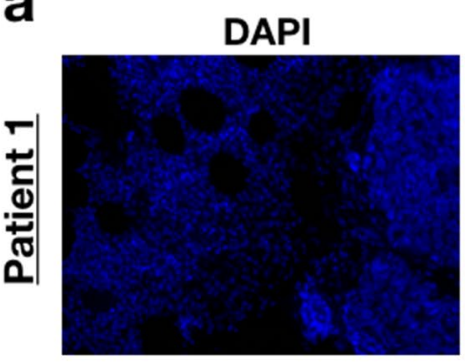

Neutrophil Elastase
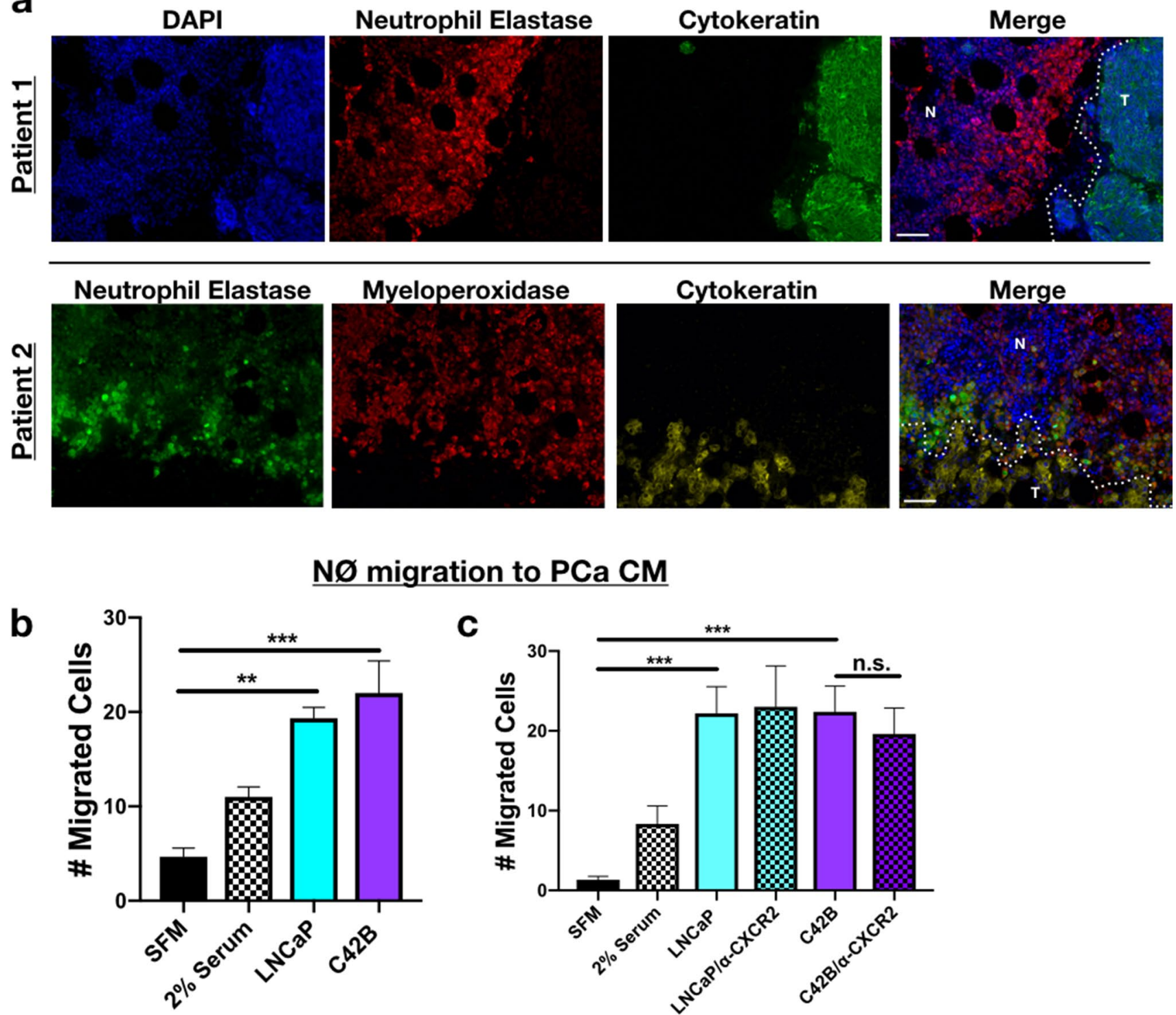

Fig. 1 PCa recruitment of neutrophils. a Representative immunofluorescence (IF) of PCa and neutrophils in bone marrow of BM-PCa patients. Top: Patient 1 -neutrophil elastase (NE; red) and epithelial marker, pan-cytokeratin (green), and nuclear marker, DAPI (blue); Bottom: Patient 2-neutrophil elastase (green), myeloperoxidase (red), cytokeratin (gold), DAPI (blue). "N" denotes normal bone marrow, "T" denotes a region of tumor in bone. Size bar $=50 \mu \mathrm{m}$. b Boyden chamber migration assay and shows number of neutrophils

that migrated through the Boyden membrane into the lower chamber. Neutrophils were allowed to migrate toward specific conditions, for $1 \mathrm{~h}$ : serum-free media, serum containing $2 \% \mathrm{FBS}$, serum-free LNCaP conditioned media (CM), and serum-free C42B CM. c Neutrophil migration assay toward $\mathrm{PCa}$ media supplemented with an antibody to mouse CXCR2 $(50 \mathrm{nM})$. Asterisks denote statistical significance $(* * p<0.01, * * * p<0.001)$

C42B-treated neutrophils produced the most ROS after $2 \mathrm{~h}$ of treatment (Supp. Figure 2B).

As an additional measure of PCa-mediated neutrophil activation, neutrophil extracellular trap (NET)-formation was examined. NETs are sticky mesh-like secretions of DNA combined with granule enzymes released by activated neutrophils to sequester and degrade bacterial pathogens and are stimulated by enzymes, myeloperoxidase and neutrophil elastase, which are also involved in neutrophil oxidative burst [18]. To examine the impact of PCa on NET secretion, primary bone marrow mouse neutrophils were incubated in prostate $\mathrm{CM}$ for $2 \mathrm{~h}$ and Sytox Green was added to the media to bind extruded DNA. Similar to the oxidative burst assay, $\mathrm{PCa}$ media significantly induced neutrophil secretion of NETs; interestingly, LNCaP and C42B media induced significantly more NETs than non-malignant prostate cells, RWPE-1 (two and threefold, respectively; $p<0.05,0.01$ ) and $\mathrm{BPH}-1$ (Fig. 2b). These findings demonstrate that $\mathrm{PCa}$ 


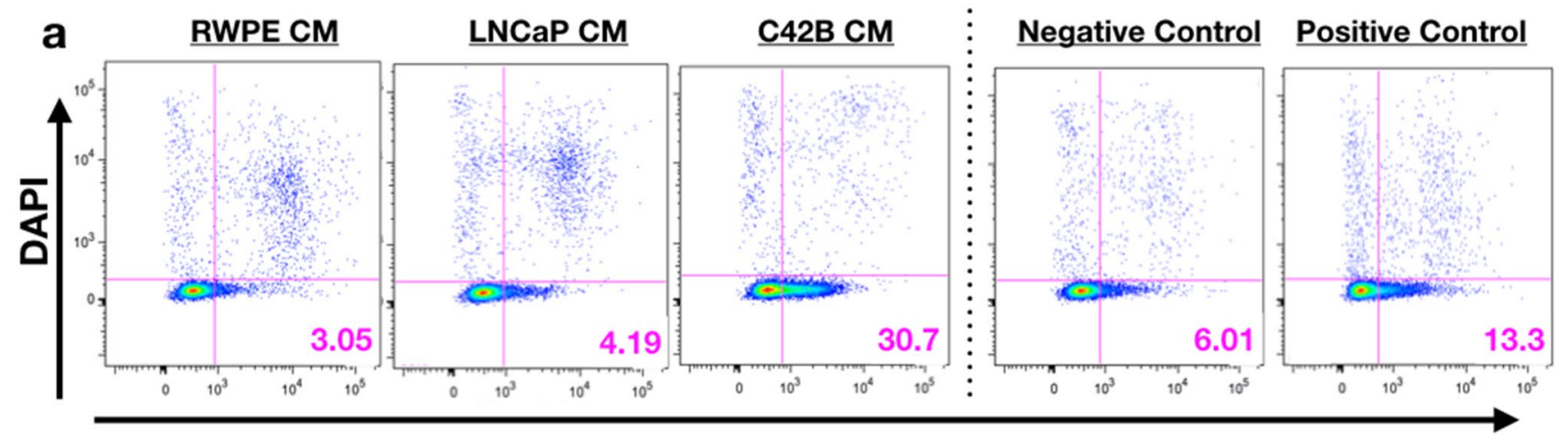

DHR 1,2,3

b

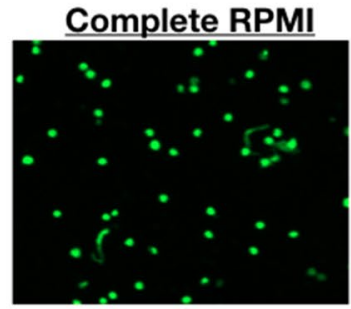

RWPE CM

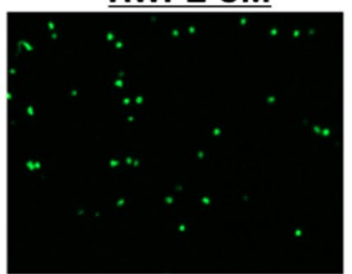

LNCaP CM

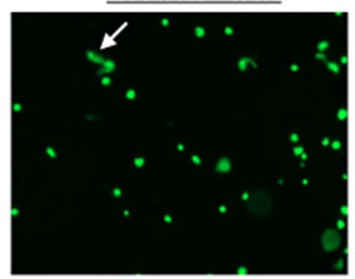

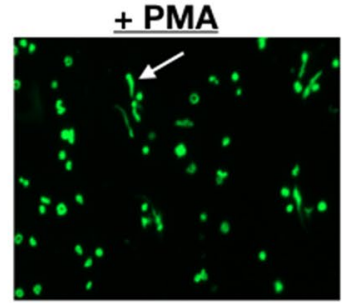

BPH1

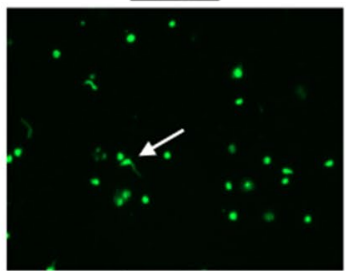

C42B CM

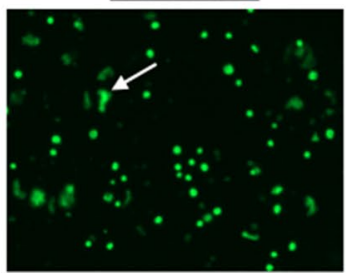

Fig. 2 PCa induces neutrophil oxidative burst and NET release. a Neutrophils incubated for $3 \mathrm{~h}$ in conditioned media (CM) from RWPE, LNCaP and C42B cell lines supplemented with $5 \mathrm{nM}$ dihydrorhodamine 123 (DHR123) and DAPI. Scatter plots show oxidative burst as expression of green fluorescence (x-axis) compared to DAPI (measure of viability) (y-axis) of CM-treated neutrophils compared to serum-free base media (negative control) and complete media, supplemented with $10 \% \mathrm{FBS}$ and $5 \mu \mathrm{g} / \mathrm{ml}$ LPS (positive control). Images

factors induce activation and, specifically, ROS and NET production of bone marrow neutrophils.

\section{Neutrophils regulate $\mathrm{PCa}$ growth in vitro independently of TGF $\beta$}

Previous evidence in other cancer types has demonstrated that cancer-induced neutrophil ROS and NETs contribute

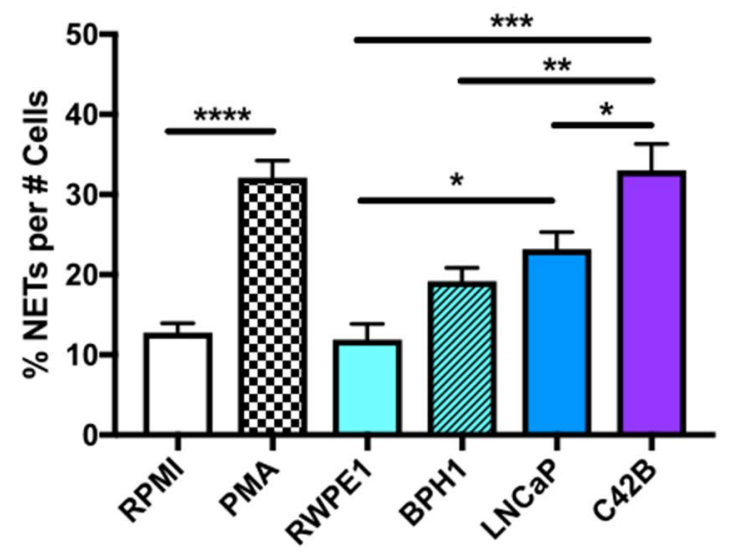

shown are representative from 1 of 4 repeat experiments. b Mouse bone marrow neutrophils incubated in CM for $2 \mathrm{~h}$ ( $n=3$ wells/media) and Sytox Green, DNA-binding dye for an additional $20 \mathrm{~min}$. Images (left) were taken at a 10x objective under a GFP laser excitation using the EVOS Auto Light Microscope. White arrows denote "string-like" DNA-containing NETs. Quantitation of NETs (graph) were defined as the percentage of NETs per total cell number per well. Asterisks denote statistical significance $(* * p<0.01, * * * p<0.001, p<0.0001)$

to tumor proliferation, demonstrated in other cancer types [19-21]. However, the consequences of neutrophil activation and direct neutrophil-BM-PCa interactions on tumor growth have not been fully explored. To test this, neutrophils were isolated from bone marrow of C $57 \mathrm{BL} / 6$ mice and cultured in direct contact at a 10:1 neutrophil/PCa ratio with LNCaP and C42B cells in vitro for $24 \mathrm{~h}$. This ratio was based on the relative numbers of neutrophil/PCa in 
preclinical bone metastasis models from our group and was determined to induce the most significant impact on $\mathrm{PCa}$ growth (data not shown). Cell counts showed that mouse neutrophils significantly reduced $\mathrm{C} 42 \mathrm{~B}$ and $\mathrm{LNCaP}$ growth compared to PCa cultured with no neutrophils (Fig. 3a). To determine whether the impact on growth was due to heightened apoptosis, $\mathrm{LNCaP}$ and $\mathrm{C} 42 \mathrm{~B}$ were pre-incubated with Caspase 3/7 Green Apoptosis Assay Reagent and then cultured in contact with neutrophils for $24 \mathrm{~h}$. As early as $6 \mathrm{~h}$ after neutrophils were added, green fluorescence (indicative of increased caspase activity) of $\mathrm{LNCaP}$ and $\mathrm{C} 42 \mathrm{~B}$ was increased $\sim$ fourfold (LNCaP: 46 with neutrophils, 11.4 LNCaP alone; C42B: 26 with neutrophils, 5.8 C42B alone).

a

Mouse Nø PCa Co-culture
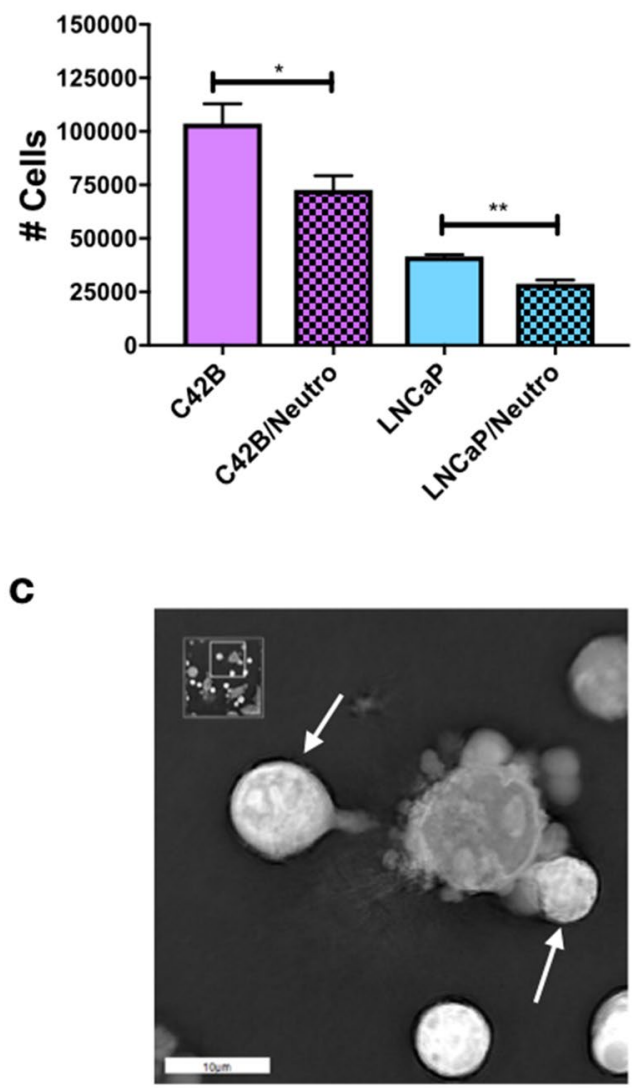

Fig. 3 Neutrophils regulate $\mathrm{PCa}$ growth in vitro. a $\mathrm{LNCaP}$ or $\mathrm{C} 42 \mathrm{~B}$ cell numbers were quantified by Trypan Blue Exclusion assay $24 \mathrm{~h}$ after direct co-culture with mouse bone marrow-derived neutrophils plated at a 10:1 ratio of neutrophil to cancer $(n=3)$. Asterisks denote statistical significance $(* p<0.05, * * p<0.01)$. b Caspase activity quantification during Incucyte S3 Live-Cell Analysis System imaging. Incucyte S3 Live-Cell Analysis System caspase-3/7 green reagent was added to neutrophil-PCa co-cultures when neutrophils were added to cancer cells. Quantification of cells was calculated based
At $24 \mathrm{~h}$, there was a significant increase in apoptosis in both $\mathrm{LNCaP}$ (at $24 \mathrm{~h}$, fivefold more than LNCaP alone, $p<0.0001$ ) and $\mathrm{C} 42 \mathrm{~B}$ (at $24 \mathrm{~h}, \sim$ threefold more than $\mathrm{C} 42 \mathrm{~B}$ alone; $p<0.0001$ ) (Fig. 3b). There were surprisingly more apoptotic $\mathrm{LNCaP}$ than $\mathrm{C} 42 \mathrm{~B}$ cells despite similar reductions in both $\mathrm{LNCaP}$ and $\mathrm{C} 42 \mathrm{~B}$ numbers observed in Trypan Blue assays and in Incucyte S3 Live-Cell Analysis System-measured cell confluency (Supp. Figure 3). Real-time holotomography microscopy (Nanolive) further supports the PCa apoptotic inducing roles of neutrophils (Fig. 3c and (Supp. Movie 1). Previous findings have demonstrated differences in mouse and human neutrophil cytotoxicity, and thus we examined the impact of human neutrophils (isolated from

b

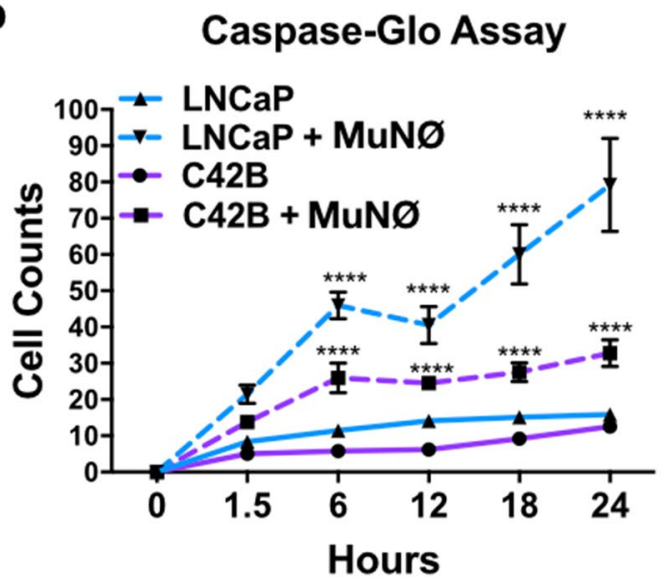

d

Human Nø PCa Co-culture

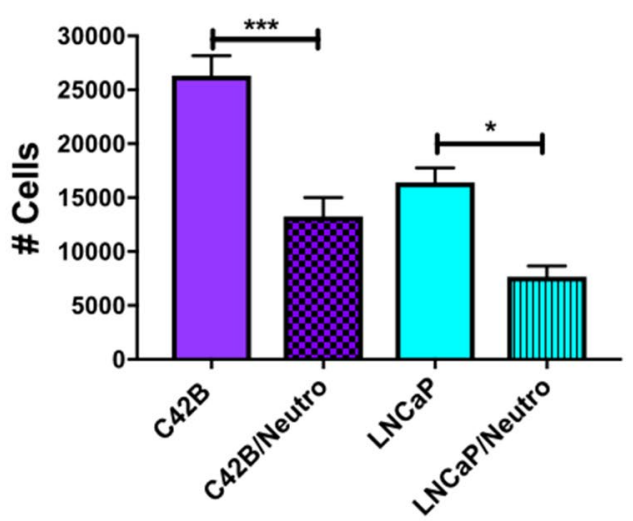

on presence of the green Caspase dye. Solid lines are PCa in culture; dashed lines are PCa cells cultured with neutrophils. Asterisks denote statistical significance $(* * * * p<0.0001)(n=3)$ for each condition. c Snapshot image of mouse neutrophils with cultured C42B cells imaged using NanoLive 3D Explorer for $1 \mathrm{~h}$. White arrows denote neutrophils surrounding a C42B cell with membrane blebbing. Asterisks denote statistical significance $(* p<0.05, * * * p<0.001)$ d Trypan Blue quantitation of $\mathrm{LNCaP}$ and $\mathrm{C} 42 \mathrm{~B} 24 \mathrm{~h}$ after culture with human bone marrow neutrophils 
human bone marrow aspirates) on $\mathrm{LNCaP}$ and $\mathrm{C} 42 \mathrm{~B}$ growth and found that human neutrophils significantly reduced both LNCaP and C42B growth by 2.5 -fold $(p<0.001 ; p<0.05)$ (Fig. 3d) demonstrating that bone marrow neutrophils are cytotoxic to BM-PCa.

Based on evidence demonstrating that TGF $\beta$ promotes the emergence of pro-tumoral neutrophils [12], we examined the impact of PCa on neutrophil TGF $\beta$ ligand and receptor gene expression. Real-time qPCR revealed that the bone metastatic C42B-derived soluble factors significantly increased mouse neutrophil expression of TGF $\beta I$, T $\beta R I$, and T $\beta$ RII by $\sim$ twofold (Supp. Figure $4 \mathrm{~A}$ ) and human neutrophil T $\beta$ RI/ALK5 expression $\sim$ fivefold $(p<0.05$ ) (Supp. Figure 4D) in support of previous findings [12]. Based on evidence showing that cancer $\mathrm{CM}$ can increase neutrophil survival [11], we examined the impact of BM-PCa and T $\beta R I$ expression on neutrophil viability. LNCaP media had the largest impact on neutrophil survival where $50 \%$ of mouse neutrophils in LNCaP media were still viable at $18 \mathrm{~h} \mathrm{com-}$ pared to SFM (i.e., baseline) and C42B ( $25 \%$ viable cells for both conditions) (Supp. Figure 4B). Likewise, human neutrophils exhibited similar responses in viability; however, luminescence of human neutrophils in PCa media increased between 24 and $48 \mathrm{~h}$ suggesting that there were more cells at those time points (Supp. Figure 4E). Inhibition of neutrophil ALK5 activity using a small molecule kinase inhibitor, RepSox, further increased neutrophil numbers and viability. ALK5 inhibition had a moderate impact on mouse neutrophil-mediated PCa apoptosis but no effect on human neutrophils (Supp. Figure 4C, F). These findings reveal that PCa-induced T $\beta R I$ is not a major mediator of neutrophilmediated PCa death but that both mouse and human neutrophils respond similarly to $\mathrm{PCa}$.

\section{Neutrophil depletion enhances growth of bone metastatic $\mathrm{PCa}$ in vivo}

To determine the effect of neutrophils in the prostate tumorbone microenvironment, luciferase-expressing C42B were injected intratibially into male SCID Beige mice. Three days after inoculation, mice were randomized based on bioluminescence signals and randomized into control (2A3 isotype control) or neutrophil-depleted (anti-Ly6G antibody; clone $1 \mathrm{~A} 8$ ) groups ( $n=5 /$ group). The $1 \mathrm{~A} 8$ antibody selectively depletes Ly $6 \mathrm{G}^{+}$cells, without depleting other Ly6Gnegative myeloid cell populations (such as macrophages) [22]. Neutrophil depletion in mouse tibia and spleen was confirmed by flow cytometry (Supp. Figure 5). Using bioluminescence as a readout for tumor growth, we observed that $1 \mathrm{~A} 8$ increased $\mathrm{C} 42 \mathrm{~B}$ growth rates over time compared to controls (Fig. 4a) (2.3-fold, $p<0.001)$. In support of this result, immunohistochemical analysis of 1A8-treated mice showed a significant increase in the proliferation marker phosphorylated histone $\mathrm{H} 3$ (pHH3) in cytokeratin-positive C42B compared to the control group (Fig. 4b). This increase in tumor volume also enhanced cancer-induced bone formation as there was no difference in the bone volume of salineinjected/“sham" limbs (Fig. 4c).

Previous studies showed that pro-tumoral neutrophils emerge in the tumor microenvironment as tumor burden increases [12]. To examine this possibility in BM-PCa, C42B cells were injected intratibially in SCID Beige mice and after 2 weeks, neutrophils were depleted using 1 A8. Similar to early stage neutrophil depletion, 1A8 increased tumor burden compared to isotype control-treated mice (Fig. 4d). Specifically, tumor burden at Day 27 was $\sim$ threefold higher in neutrophil-depleted mice compared to isotype control (bioluminescence: $8.9 \times 10^{6}(1 \mathrm{~A} 8)$ vs. $2.8 \times 10^{6}$ (control)) suggesting that neutrophils in the tumor-bone microenvironment are predominantly anti-tumoral and that depletion of these populations accommodates BM-PCa growth in bone.

For comparison in another bone metastatic PCa model (i.e., prostate tumor-derived cells that grow in bone), we examined the importance of neutrophils in the growth of PAIII rat adenocarcinoma, which have been shown to induce osteolytic and osteogenic bone lesions in vivo. Similar to our findings with the C42B model, depletion of neutrophils in the PAIII intratibial model increased PAIII growth in bone which showed a 3.5-fold increase in luminescence of neutrophil-depleted mice at Day 8 (Fig. 5a, top). We demonstrated that these findings were not simply due to the PCa "filling in" the additional bone marrow space provided by the neutrophil depletion as previously shown [23], by depleting neutrophils from the intratibial model of LNCaP, cells considered poorly metastatic since they do not typically grow in bone [24]. Despite the ability of neutrophils to kill LNCaP in vitro, $\mathrm{LNCaP}$ did not grow in bone even in the absence of neutrophils (Fig. 5a, bottom) in the bone marrow space.

Although these findings suggest that neutrophils in the tumor-bone environment are predominantly cytotoxic, we next examined the impact of BM-PCa on neutrophil function throughout tumor progression in bone. To do this, C42B cells were injected intratibially in SCID Beige mice and bone marrow neutrophils/tumor-associated neutrophils (TANs) were isolated from bone at Weeks 1, 2, and 4 post- $\mathrm{C} 42 \mathrm{~B}$ injection for the following functional analyses: impact on PCa growth in PCa-neutrophil co-culture assays, neutrophil viability, NET formation and T cell suppression. TAN function was compared to neutrophils isolated from mice injected intratibially with saline. As previously seen, both control neutrophils and TANs inhibited LNCaP and C42B growth at Weeks 1 and 2 ( 2.5-fold, $~ 30 \%$ reduction in growth); however, TANs isolated from marrow 4 weeks post-injection did not induce PCa cell death and appeared to have no impact on PCa growth (Fig. 5b). Further, Week 4 
a C42B Intratibial Model

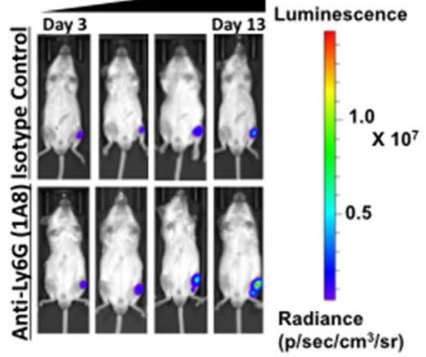

Bioluminescence

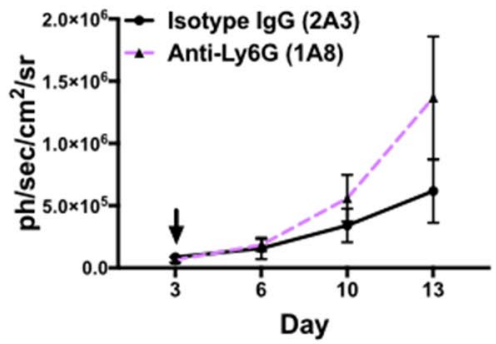

Change in Tumor Burden

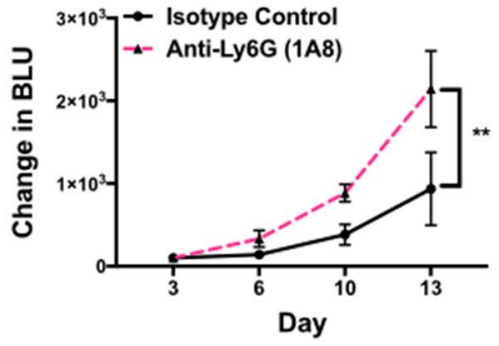

b

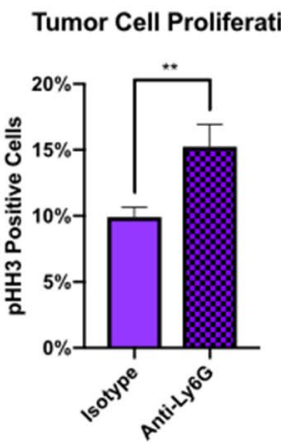

pHH3
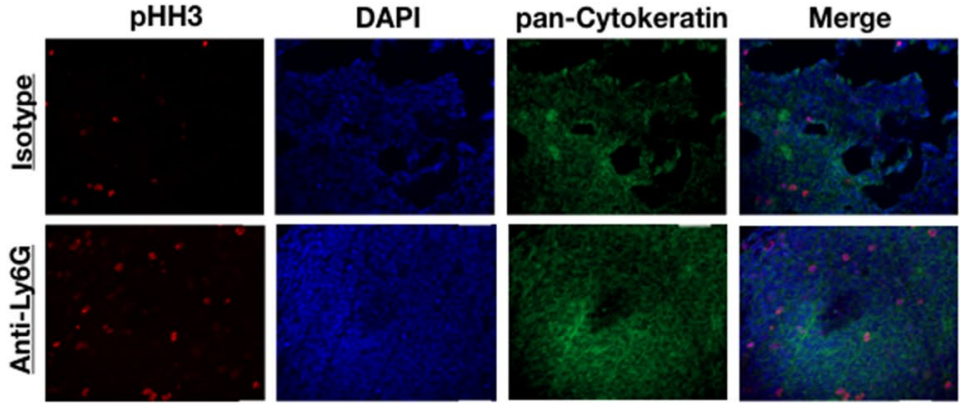

C

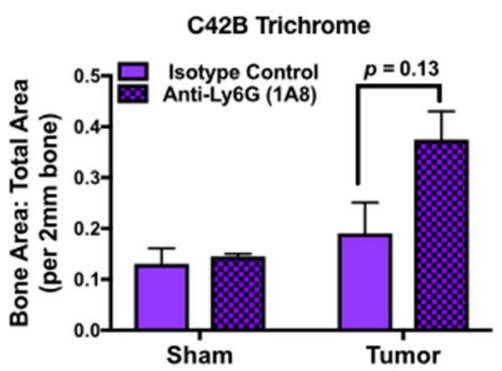

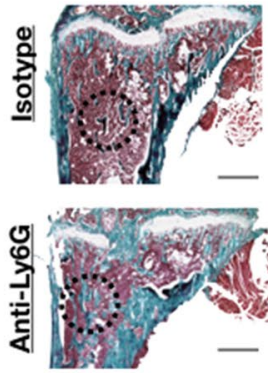

Fig. 4 Neutrophils mediate growth of bone metastatic PCa in vivo. a Luciferase-expressing C42B cells were injected intratibially into SCID Beige mice. Representative images, quantitation of bioluminescence (left graph), and change in tumor bioluminescence compared to start of study (right graph) of C42B ( $n=5 /$ group) treated with isotype control or anti-Ly6G 1A8 antibody 3 days after inoculation. Arrow denotes start of neutrophil depletion. b Quantitation (graph on left) and representative IF images (right) of C42B (denoted by cytokeratin (green)) expression of phosphorylated Histone H3 (red) in bone. Nuclei are stained with DAPI (blue). Size bar $=20 \mu \mathrm{m}$. Asterisks

TANs showed different functional characteristics compared to Week 1 and 2 TANs and exhibited prolonged cell viability (40\% viable cells at $24 \mathrm{~h}$ and $20 \%$ viable at $48 \mathrm{~h} ; p<0.001$ ) compared to complete cell death of control neutrophils and Week 1 and 2 TANs by 24 h (Supp. Figure 6A). Additionally, Week 4 TANs produced significantly less NETs implicating reduced activation (Supp. Figure 6B).

Finally, we examined the $\mathrm{T}$ cell suppressive properties of control bone marrow neutrophils compared to TANs at Weeks 1 and 4 post-tumor injection. Naïve $\mathrm{CD}^{+}{ }^{+} \mathrm{T}$ cells were isolated from spleen of C57BL/6 mice and cultured directly with control neutrophils or TANs along with CD3

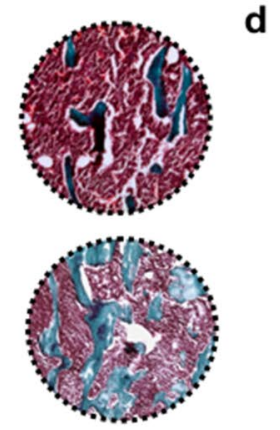

denote statistical significance $(* * p<0.01)$ c Trichrome quantitation of saline-injected (sham) and tumor-inoculated tibia of isotype vs. anti-Ly6G-treated mice in representative graph (left); representative images (left) show Trichrome stain of tumor tibia with magnified inset. Type I collagen/bone is stained blue. Size bar $=500 \mu \mathrm{m}$. d Bioluminescence of luciferase-expressing C42B cells. C42B-luc cells were injected intratibially into SCID Beige mice ( $n=5 /$ group) and neutrophil depletion (via anti-Ly6G (1A8)) or isotype control treatments were started 2 weeks post-tumor inoculation (at arrow), denoted as late depletion

and CD28 Dynabeads to stimulate T cell proliferation. Surprisingly, both tumor-naïve control neutrophils and TANs were significantly suppressive of $\mathrm{T}$ cell proliferation such that there were fewer proliferating/more non-proliferating $\mathrm{T}$ cells compared to the positive control T cells with no neutrophils ( $\sim$ two- to threefold difference; $p<0.01$ ). However, by Week 4 post-injection, TANs were $\sim$ twofold less suppressive of $\mathrm{T}$ cells than the control neutrophils (Supp. Figure 6C). These findings revealed that: (1) bone marrow neutrophils cytotoxic to $\mathrm{PCa}$ are also suppressive of $\mathrm{T}$ cell proliferation and (2) that TANs in the tumor-bone microenvironment are functionally altered throughout tumor progression. 

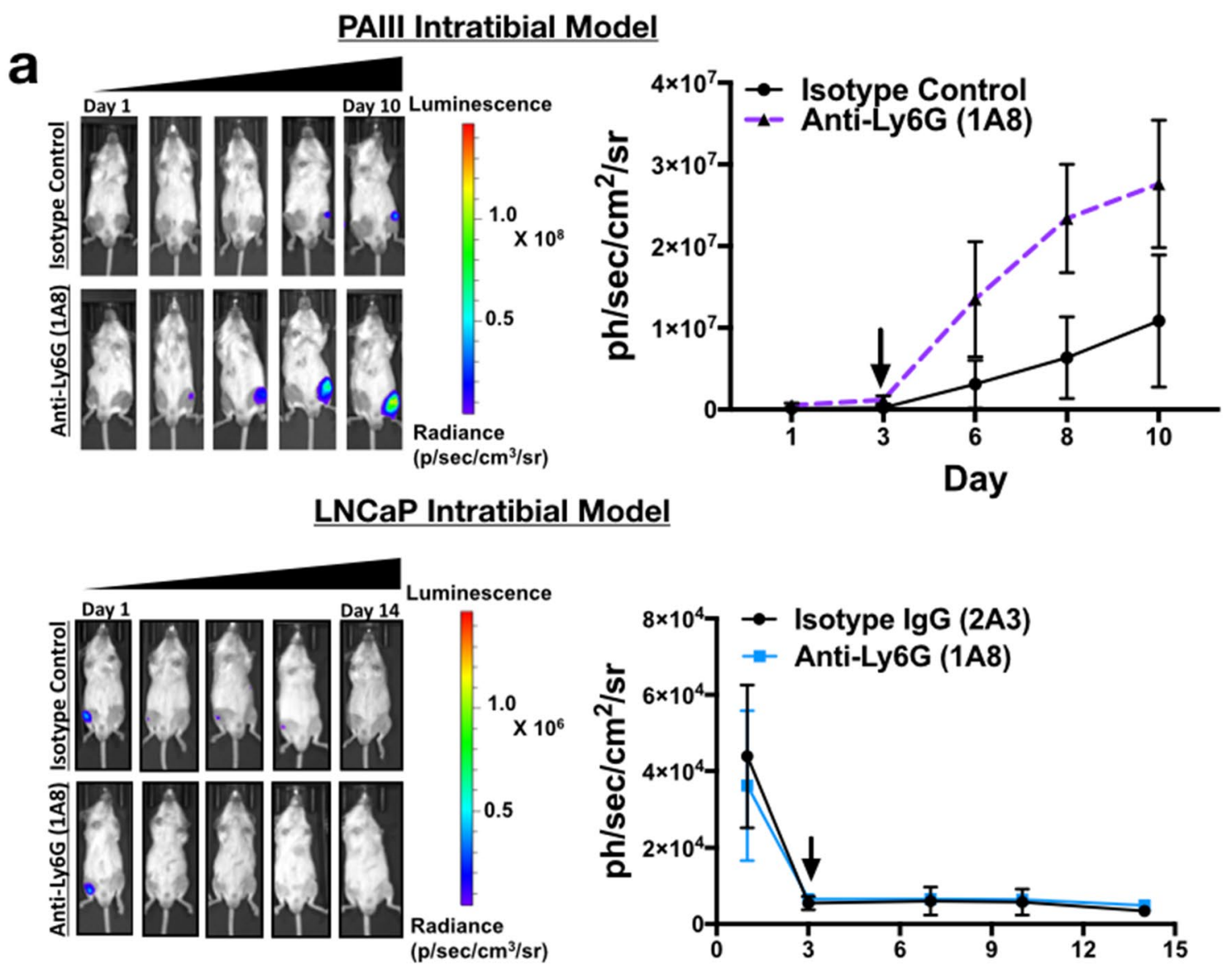

b
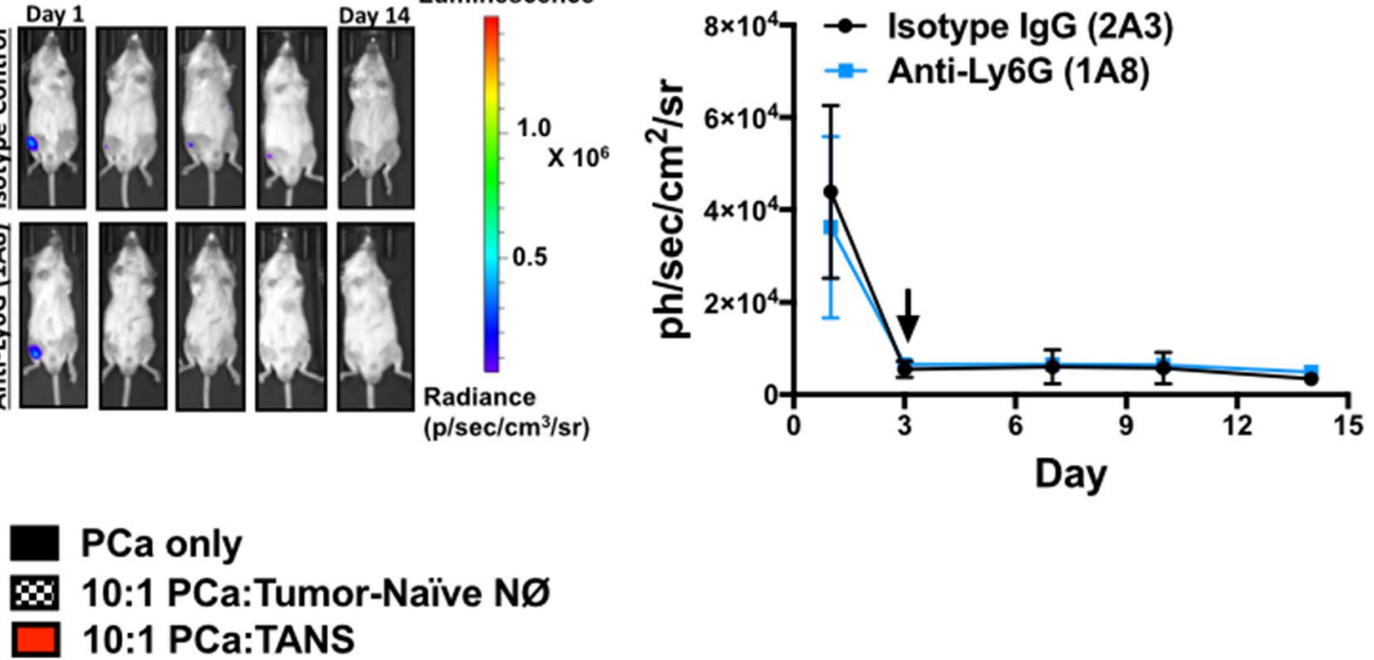

Week 1
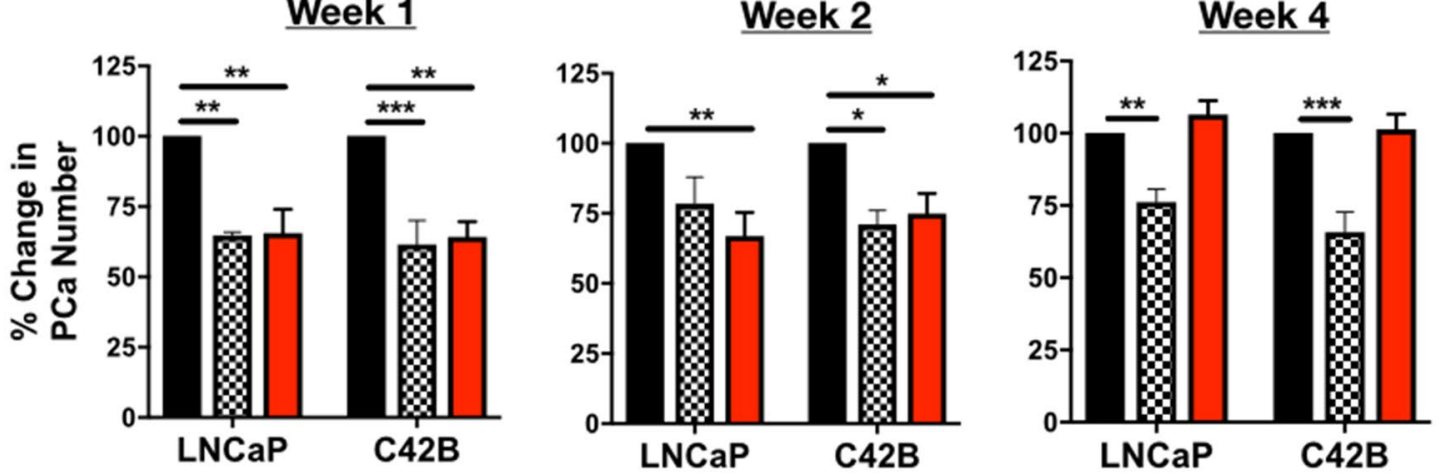

Fig. 5 Neutrophil depletion is permissive for bone metastatic but not non-metastatic prostate cancer growth in bone but lose cytotoxicity with tumor progression. a Top, PAIII cells were injected intratibially into SCID Beige mice ( $n=5 /$ group) and treated with anti-Ly6G (1A8) or isotype control antibody. Bioluminescence is shown in representative images (left). Graph represents change in bioluminescence/growth rate from day of neutrophil depletion start at Day 3 (right). Arrow in graph denotes start of neutrophil depletion. Bottom, bioluminescence of luciferase-expressing LNCaP cells. LNCaP-luc were injected intratibially into SCID Beige mice ( $n=5 /$ group) and neutrophil depletion started 3 days post-tumor inoculation. Arrow indicates start of treatment. b C42B cells (or saline as a control) were injected intratibially into male SCID Beige mice and mice euthanized at Weeks 1, 2 and 4 post-injection for isolation of bone neutrophils. Control neutrophils (from saline-injected tibia; denoted as NØs) and tumor-associated neutrophils (TANs) were cultured ex vivo with LNCaP and C42B cell lines for $24 \mathrm{~h}$ and counted via Trypan Blue Exclusion assay. Graphs show percent change in cell number after $24 \mathrm{~h}$ of culture with neutrophils. Asterisks denote statistical significance $(* p<0.05, * * p<0.01, * * * p<0.001)$ 
Collectively, these results demonstrate that bone marrow neutrophils suppress PCa growth in vivo and in vitro and, specifically, metastatic PCa is able to grow in bone, in part, by evading neutrophil-mediated cell killing.

\section{Bone-resident neutrophils induce $\mathrm{PCa}$ apoptosis via inhibition of Stat signaling}

We next considered the molecular pathways being targeted by neutrophils that could promote PCa cell death. Unlike the effects noted with T $\beta$ RI inhibition on neutrophil viability, pre-incubating neutrophils with the T $\beta$ RI inhibitor RepSox and subsequent direct co-culture with PCa revealed no impact on PCa viability (Supp. Figure 4B and 4E). We therefore employed a more global approach. Using a receptor tyrosine kinase phosphorylation array, we examined the status of LNCaP and C42B kinase signaling in response to neutrophils and observed a significant reduction in the phosphorylation of several kinases important for prostate cancer cell survival (Fig. 6a and Supp. Figure 7A). Specifically, to distinguish between mouse and human proteins, neutrophils isolated from mouse bone marrow were cultured with the human PCa cells, protein was isolated from the PCa cells, and specific protein changes were validated in human neutrophil co-cultures, using human neutrophils isolated from bone marrow in culture with the human PCa cell lines. Phosphorylation of the non-receptor kinase Src was significantly reduced 5.5-fold in $\mathrm{C} 42 \mathrm{~B}$ cultured with neutrophils (0.11 vs. $0.02 ; p<0.0001)$, compared to neutrophil-treated LNCaP (no change in Src). Additionally, phosphorylation of Src downstream target, STAT5, was significantly inhibited in C42B (0.19 vs. 0.09; $p<0.0001)$. Interestingly, mouse neutrophils significantly inhibited Stat 3 phosphorylation in LNCaP, whereas Stat3 was unaffected in C42B suggesting that Stat pathways may be critical mediators of $\mathrm{BM}-\mathrm{PCa}$ growth in bone (Fig. 6b). To determine whether STAT5 phosphorylation is related to neutrophil direct contact with $\mathrm{PCa}$ (and to confirm that the STAT changes were not species specific), we isolated neutrophils from human bone marrow and performed direct and indirect (using modified Boyden Chamber) co-culture assays. Although neutrophils reduced PCa numbers in indirect co-culture, total and phosphorylated STAT5 was inhibited more with direct compared to indirect co-culture conditions (Supp. Figure 7B). In support of these findings, IF of neutrophil-depleted C42B intratibial tumors revealed significantly more STAT5 expression compared to isotype control-treated mice (Fig. 6c). Interestingly, human neutrophils had no impact on the growth of PC3 PCa, which are STAT5 negative compared to STAT5-expressing PAIII cells (Fig. 6d).

Previously STAT5 has been shown to promote metastatic prostate cancer growth, such that STAT5 inhibition results in reduced growth of BM-PCa cells [25]. To determine whether
STAT5 is important for neutrophil-induced PCa death, we reduced C42B expression of STAT5 via shRNA. Based on qPCR analysis of $\mathrm{C} 42 \mathrm{~B}$ gene expression after neutrophil co-culture which showed a significant reduction in STAT5A compared to STAT5B (Supp. Figure 8A), we focused on STAT5A inhibition. However, knockdown of STAT5A gene expression via a STAT5A shRNA also reduced gene expression of STAT5B (Supp Figs. 8B, C), which is 92\% homologous to STAT5A. Mouse bone marrow neutrophils had no impact on C42B with reduced STAT5 expression (STAT5 KD C42B) compared to control C42B e.g., expressing a non-targeted scrambled sequence shRNA (Fig. 6e). Despite overlap in STAT5A/B targeting, there was little impact on STAT3, another STAT targeted by mouse neutrophils in LNCaP (Fig. 6a and Supp. Figure 8D), demonstrating the specificity of the gene knockdown to STAT5. These findings revealed that neutrophil-mediated PCa apoptosis is dependent on PCa STAT5 expression. Further analysis will be required to define the specific mechanisms involved in neutrophil-mediated STAT5 inhibition.

\section{Discussion}

The majority of PCa-associated deaths are due to the development of advanced stage castration-resistant disease, which typically progresses to incurable bone metastatic PCa (BM-PCa). Current standard immunotherapies targeting $\mathrm{T}$ cell activation (e.g., checkpoint inhibition blockade) have been beneficial for some advanced stage cancers but have been less reliable for patients with bone metastatic $\mathrm{PCa}$ ranging from complete response to no response at all $[26,27]$. However, given the abundance of immune cells in the BM-PCa bone microenvironment, there is a significant need for defining the role of immune populations in regards to disease progression. With the abundance of neutrophils in the bone microenvironment and existing evidence of TGF $\beta$ driving pro-tumoral neutrophil function, we hypothesized that tumor-associated neutrophils in bone contribute to $\mathrm{PCa}$ progression. This hypothesis was supported by evidence of neutrophil recruitment toward $\mathrm{PCa}$ media and patient bone biopsies showing regions of neutrophil localization to the tumor interface and PCa recruitment of bone marrow-derived neutrophils in vitro (Fig. 1). Surprisingly, our studies demonstrated that $\mathrm{PCa}$ activates bone-derived neutrophils, which are cytotoxic to $\mathrm{PCa}$ and directly induce $\mathrm{BM}-\mathrm{PCa}$ apoptosis via targeting of STAT5. These are the first studies, to date, to show the impact of direct neutrophil-PCa interactions on metastatic growth in bone.

Our findings showed that neutrophils directly inhibited STAT5 expression in C42B, which appears to be necessary for neutrophil-mediated PCa apoptosis. Recent studies showed that STAT5 is a mediator of growth and invasion of 

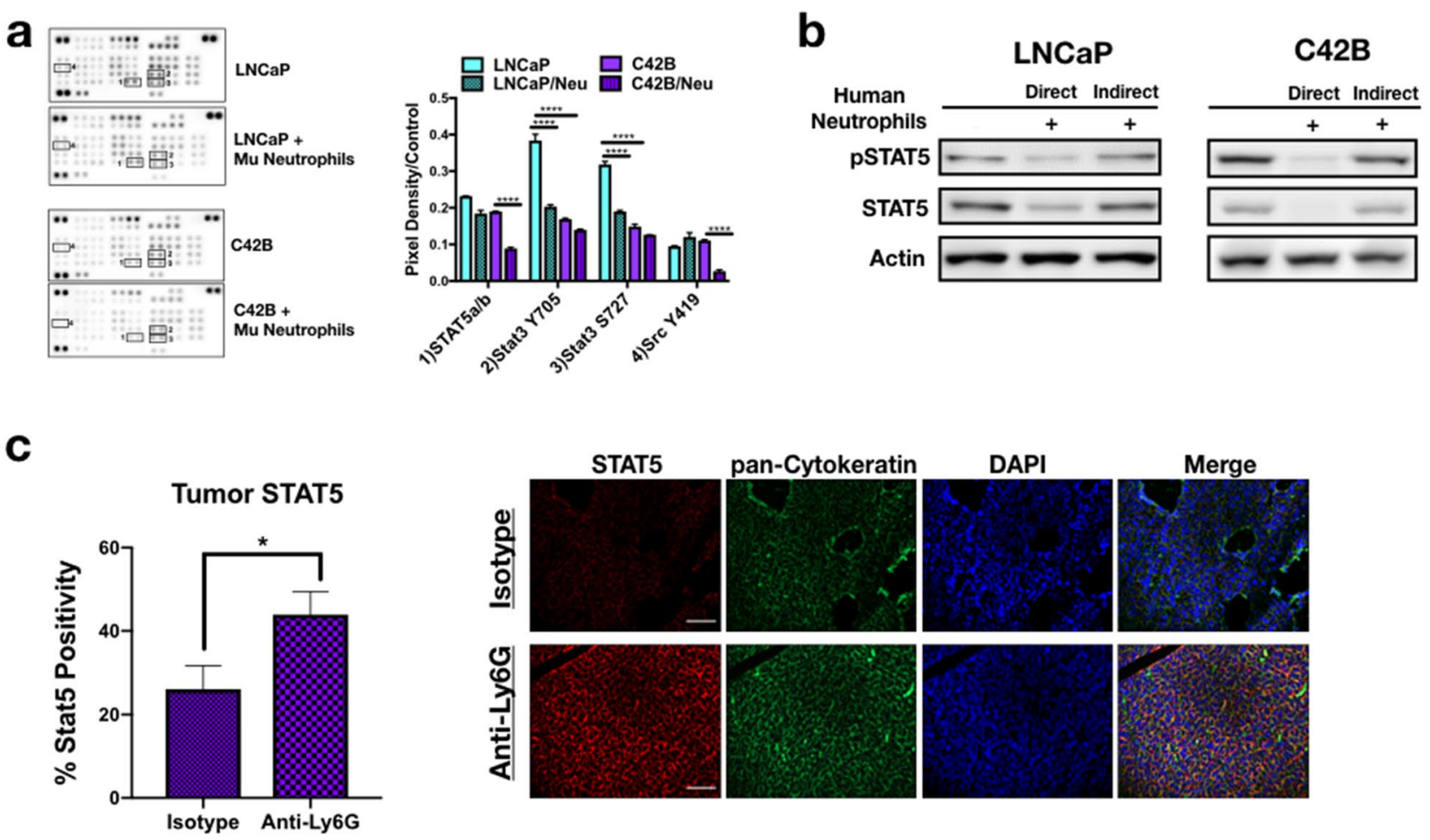

d

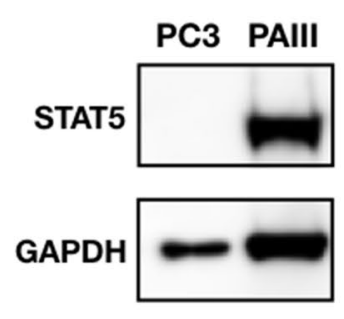

e

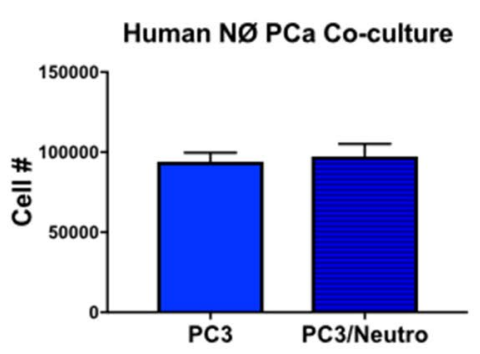

C42B STAT5A KD:NØ Co-culture

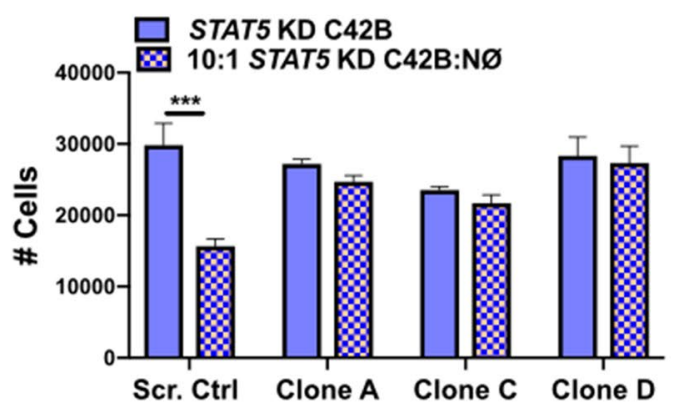

Fig. 6 Neutrophils induce PCa death via inhibition of STAT5. a Phospho-kinase protein array of $\mathrm{LNCaP}$ and $\mathrm{C} 42 \mathrm{~B}$ cells cultured alone or with mouse bone marrow-derived neutrophils (denoted as $\mathrm{Mu}$ neutrophils). Boxes correspond to the genes labeled in. Three samples of each condition were pooled, and $100 \mu \mathrm{g}$ of protein loaded onto the array. Proteins are displayed in duplicate. Average dot pixel density was averaged per protein target, background density subtracted, and normalized to the reference control per blot. Densitometry analysis was performed using Image $\mathbf{J}$ software. Graph represents pixel density quantitation of pSTAT5, pSTAT3, and pSrc. Asterisks denote statistical significance $(* * * * p<0.0001)$. b STAT5 western blot of whole-cell PCa ( $30 \mu \mathrm{g}$ protein) lysates from direct and indirect co-cultures of human neutrophils with $\mathrm{LNCaP}$ (left) and C42B (right) cells. For indirect co-cultures, modified Boyden chamber assay

bone metastatic PCa where siRNA-mediated STAT5 inhibition significantly increased PCa Caspase 7 expression and apoptosis [25, 28]. Additional studies showed that STAT5 expression is increased in aggressive prostate cancer and was utilized. c Immunofluorescence quantitation is the percentage of STAT5-positive C42B per total cell number (left) and representative images (right) of Total STAT5 (red) in C42B bone metastases $(n=5)$. C42B tumor cells in bone are denoted by cytokeratin (green), and nuclei are stained by DAPI (blue). Size bar $=20 \mu \mathrm{m}$; asterisk denotes statistical significance $(* p<0.05)$. d Left, Western blot analysis of Stat5 in PC3M compared to PAIII. Right, PC3M cell counts $24 \mathrm{~h}$ after direct co-culture with human bone marrow neutrophils. e Direct co-culture of bone-derived mouse neutrophils and C42B cells expressing: a non-targeted scrambled sequence shRNA (Scr. Ctrl) or STAT5A shRNA plasmids (denoted STAT5 knockdown (KD) clones A, C, and D). Graph shows the total number of C42B cells $24 \mathrm{~h}$ after co-culture measured by Trypan Blue Exclusion assay. Asterisk denotes statistical significance $(* * * p<0.001)$

inhibition of STAT5 was able to sensitize BM-PCa xenografts to radiation through blockade of STAT5-mediated DNA repair $[29,30]$. Our studies revealed that neutrophilmediated PCa death is due to specific inhibition of STAT5 
expression supporting prior evidence that STAT5 is a potential target for preventing progression of aggressive, metastatic PCa. However, STAT5 is a critical factor for neutrophil function and T cell differentiation [31, 32]; thus, STAT5targeting of BM-PCa would require tumor-specific delivery to prevent negative effects on the surrounding immune cells.

It is possible that BM-PCa tumors in bone display heterogeneous STAT5 expression, as previously identified in primary PCa tumors with intratumor variability in STAT5 protein levels [33], such that neutrophil targeting would inevitably result in the emergence of a neutrophil-resistant population, similar to acquired drug resistance. There is an additional possibility that neutrophil-resistant PCa may alter long-term neutrophil function. A similar scenario was recently demonstrated in a study of metastatic breast cancer in which IL11-expressing breast cancer cells induced a specific pro-tumoral neutrophil gene signature compared to IL11-null breast tumors which recruited tumor-suppressive neutrophils [34]. As seen with the emergence of acquired drug resistance, future studies are needed to identify specific mechanisms associated with PCa resistance to neutrophil cytotoxicity and the impact of neutrophils on heterogeneous STAT5-expressing BM-PCa tumors.

There is little to no understanding of the contribution of neutrophil/neutrophil precursor populations to tumor growth in the PCa tumor-bone microenvironment. Previously, two different neutrophil subpopulations were identified in lung tumor tissue, namely anti-tumorigenic, N1, and pro-tumorigenic, N2 PMNs, the latter accumulated as a result of increased TGF $\beta$ in the tumor microenvironment [12]. These descriptors have been expanded based upon context-specific neutrophil roles $[21,35]$. We found that, although BM-PCa increased neutrophil expression of T $\beta R I$, this increase had little impact on neutrophil-mediated PCa apoptosis despite increasing neutrophil viability. Depletion of neutrophils in an established bone tumor compared to post-dissemination (recapitulated by depletion 3 days after tumor inoculation) resulted in accelerated tumor growth suggesting that neutrophils in the tumor-bone environment are initially cytotoxic and anti-tumoral. This was further shown by neutrophil activation (i.e., oxidative burst and NET formation) in response to PCa media. However, longitudinal evaluation of neutrophils isolated from the bone marrow throughout tumor progression revealed that bone TANs lose their cytotoxic potential as the tumor progressed. This change in function was associated with reduced neutrophil maturation, evidenced by prolonged viability (characteristic of pre-mitotic neutrophils) and reduced activation/NET formation, characteristics linked to tumor-associated immature neutrophils/granulocytic precursors [36]. Our findings suggest that TANs in the tumor-bone microenvironment initially exhibit characteristics associated with both cytotoxic/N1and granulocytic MDSCs (G-MDSCs) [37] but are functionally altered as the tumor grows via undetermined mechanisms. These findings reveal a need for full characterization of specific neutrophil populations associated with PCa killing within the prostate tumor-bone microenvironment to identify methods to prevent PCa growth by enhancing neutrophil cytotoxicity and/ or prevent loss of neutrophil anti-tumoral function.

In these studies, we utilized immunocompromised mice for investigation of human PCa growth in bone. Despite differences that exist between human and mouse neutrophils, we demonstrated that both human and mouse bone marrowderived neutrophils were cytotoxic to human PCa cells and that our data were not based upon species-specific neutrophil functions. Additionally, we found that bone marrow neutrophils inhibit tumor growth in the bone microenvironment, independently of $\mathrm{T}$ cell immunosuppressive functions. Interestingly, cytotoxic TANs isolated from the prostate tumorbone microenvironment were significantly suppressive of $\mathrm{CD} 4+\mathrm{T}$ cell proliferation. Although previous studies have shown that neutrophils or granulocytic MDSCs recruited to the primary tumor are associated with increased tumor growth, we present evidence that TANs in the tumor-bone microenvironment can be both tumor-inhibitory and immunosuppressive. In support of our findings, a recent study revealed that mature peripheral blood neutrophils displaying classical cytotoxic effector functions were significantly suppressive of $\mathrm{T}$ cell proliferation compared to non-cytotoxic, immature neutrophils [38] demonstrating that cytotoxic neutrophils can suppress $\mathrm{T}$ cells. In our studies, both tumor-naïve neutrophils and TANs isolated from the prostate tumor-bone environment were $\mathrm{CD} 11 \mathrm{~b}^{+} / \mathrm{Gr}-1^{\mathrm{hi}}$, markers associated with G-MDSCs in the tumor microenvironment [39], that displayed cytotoxic effects against PCa. Importantly, therapeutic depletion of these cells may be counterintuitive against treatment of BM-PCa. These findings emphasize the need for characterization of context-specific neutrophil functions and cell surface markers to identify specific effector populations in the tumor microenvironment. Understanding the direct functions of TANs in the bone marrow and their interaction with other immune populations are critical for developing myeloid-targeted therapies to treat bone metastasis.

These studies are the first, to date, to examine direct interactions of BM-PCa and neutrophils, which revealed a role for neutrophils in BM-PCa progression. For prostate cancer patients with metastatic disease, an elevated neutrophil-to-lymphocyte ratio (NLR) has been associated with poor prognosis for survival [40]. Although overall NLR has been shown to be important for disease stratification, it remains unclear whether circulating neutrophils recapitulate neutrophil function in the tumor-bone microenvironment. It is possible that neutrophil mobilization into blood could be induced by PCa in bone and this could be a mechanism of PCa to evade neutrophil-mediated death; however, this 
possibility has not yet been examined. Our findings demonstrated that bone marrow neutrophils initially will induce death of disseminated PCa cells and lose this impact as the tumor progresses. These results implicate that there may be a specific therapeutic window after PCa dissemination into the bone, where an enhanced neutrophil cytotoxicity could be used to prevent tumor growth. This also suggests that therapies that induce neutropenia, such as chemotherapy, may contribute to disseminated tumor growth in bone. In support of this, a previous study showed that cyclophosphamide enhanced PCa growth in bone via impact on the bone stromal cells, though neutrophils were not investigated [41]. Further, BM-PCa patients typically receive androgen deprivation therapy which could impact immune cell differentiation. Specifically, loss of androgen receptor (AR) inhibits precursor neutrophil proliferation and maturation [42] and it is unclear how this would impact neutrophil function in a clinical setting.

The findings from this study suggest that harnessing neutrophil cytotoxicity in bone by regulating neutrophil maturation and mobilization could prevent $\mathrm{PCa}$ growth in bone. For example, granulocyte colony-stimulating factor (G-CSF) is a primary regulator of neutrophil proliferation and differentiation and is often given to cancer patients to replenish the neutrophil population after chemotherapy-related neutropenia [43]. G-CSF mimetics are currently in clinical trial in combination with chemotherapy and could be considered for treatment of BM-PCa [44]. Although we did not examine the role of androgen deprivation therapy on neutrophil function in the prostate tumor-bone microenvironment, ongoing studies are examining the impact of standard of care BM-PCa therapies in the immune-bone microenvironment.

While other preclinical PCa studies highlighted that neutrophils contribute to the progression of prostate tumors, there remains a significant gap in the understanding of neutrophil function in the context of the tumor-bone microenvironment and BM-PCa. Although BM-PCa survival and proliferation in bone is heavily dependent on interactions with and activation of surrounding bone stromal cells, such as osteoclasts and osteoblasts; this is the first study to demonstrate a protective role for neutrophils against $\mathrm{BM}-\mathrm{PCa}$ via STAT5-mediated PCa death, a phenotype that is altered as the tumor progresses. In summary, neutrophils in bone play a significant role in regulating growth of disseminated $\mathrm{PCa}$ and harnessing neutrophil cytotoxic activity in bone could present a novel immunotherapeutic strategy for treating BM-PCa.

Acknowledgements The authors thank Dr. Conor Lynch for kindly providing all cell lines used in this manuscript. The authors thank the patients and their families who generously donated tumor tissue, and Celestia Higano, Evan Yu, Pete Nelson, Heather Cheng, Elahe Mostaghel, Bruce Montgomery, Mike Schweizer, Lawrence True, Dan Lin, Eva Corey, and the rapid autopsy teams for their contributions to the University of Washington Prostate Cancer Donor Rapid Autopsy Program.

Author Contributions DG and LMC designed research. DG, TKe, MA, AC, GFW, YY, JSF, CH, and LMC performed research and also analyzed the data. AC, $\mathrm{KS}, \mathrm{TKi}$, and $\mathrm{CM}$ contributed reagents and analysis tools. DG and LMC wrote the paper. All authors edited and approved the submission of this work.

Funding LMC was supported by a postdoctoral fellowship (PF-13-17501-CSM) and a Research Scholar Grant (RSG-19-127-01-CSM) from the American Cancer Society. AJC was supported by funding from the National Institute of Health (R00HL123471). TKi was supported by funding from the National Institutes of Health/National Institute of Allergy and Infectious Disease Grant P01 AI083211 (Project 4). This work was supported in part by the Flow Cytometry, Small Animal Imaging Laboratory, and Microscopy cores at the H. Lee Moffitt Cancer Center and Research Institute funded in part by Moffitt's Cancer Center Support Grant (P30-CA076292). Tissue acquisition in the University of Washington Prostate Cancer Donor Rapid Autopsy Program was supported by the Pacific Northwest Prostate Cancer SPORE (P50CA97186).

\section{Compliance with ethical standards}

Conflict of interest The authors declare that they have no conflict of interest.

Animal source Fox Chase SCID Beige mice were obtained from Charles River Laboratory (USA). C57Bl/6 J mice were obtained from Jackson Laboratories (Bar Harbor, ME, USA). Animals were maintained on a 12-h light/dark schedule and had free access to laboratory chow (Teklad LM 485) and water.

Cell line authentication All human cell lines used in this manuscript were processed by Genetica Cell Line Testing (a LabCorp brand; Burlington, NC, USA) for authentication testing using analytical procedures for DNA extraction, polymerase chain reaction (PCR) and capillary electrophoresis on a 3130xl genetic analyzer (Applied Biosystems). The thirteen core CODIS short tandem repeat (STR) loci plus PENTA $\mathrm{E}$ and PENTA D, and the gender-determining locus, amelogenin, were analyzed using the commercially available PowerPlex ${ }^{\circledR} 16 \mathrm{HS}$ amplification kit (Promega Corporation; mouse marker included) and GeneMapper ID v3.2.1 software (Applied Biosystems). Appropriate positive and negative controls were used concurrently throughout the analysis. Authentication of each cell line was confirmed by entering the STR DNA profile of each tested cell line into known repository cell line databases (i.e. ATCC, DSMZ); authentication is defined as having a percent match with the reference STR profile at or above $80 \%$ when using the ANSI/ATCC guidelines (ASN-0002-2011) OR having a "unique" STR DNA profile (no matches found) for "in-house" cell lines not distributed by any cell line repository.

Ethical approval and ethical standards Patient prostate cancer samples were obtained from patients who died of metastatic CRPC and who signed written informed consent under the aegis of the Prostate Cancer Donor Program at the University of Washington [45]. The study was approved by the institutional review board at the University of Washington. Human bone marrow was purchased from the Lonza Walkersville, Inc. operates a Research Bone Marrow Donor Program (Walkersville, MD, USA) to recruit, screen, test, evaluate and select donors, who sign informed consent, for collection of bone marrow samples for research use. This Donor Program is currently approved, has been approved for over 10 years, and is submitted for annual approval 
by a commercial institutional review board. All applicable international, national, and/or institutional guidelines for the care and use of animals were followed. All procedures performed in studies involving animals were in accordance with the ethical standards of the University of Nebraska Medical Center. The study was approved by the Institutional Committee for the Use and Care of Laboratory Animals of University of Nebraska Medical Center (Omaha, NE, USA). Animal research approval number: 17-113-12.

Open Access This article is licensed under a Creative Commons Attribution 4.0 International License, which permits use, sharing, adaptation, distribution and reproduction in any medium or format, as long as you give appropriate credit to the original author(s) and the source, provide a link to the Creative Commons licence, and indicate if changes were made. The images or other third party material in this article are included in the article's Creative Commons licence, unless indicated otherwise in a credit line to the material. If material is not included in the article's Creative Commons licence and your intended use is not permitted by statutory regulation or exceeds the permitted use, you will need to obtain permission directly from the copyright holder. To view a copy of this licence, visit http://creativecommons.org/licenses/by/4.0/.

\section{References}

1. Koo KC et al (2015) Prognostic impacts of metastatic site and pain on progression to castrate resistance and mortality in patients with metastatic prostate cancer. Yonsei Med J 56(5):1206-1212

2. Frieling JS et al (2015) Current and emerging therapies for bone metastatic castration-resistant prostate cancer. Cancer Control 22(1):109-120

3. Juarez P, Guise TA (2010) Tgf-Beta pathway as a therapeutic target in bone metastases. Curr Pharm Des 16(11):1301-1312

4. Yu C et al (2012) Prostate cancer and parasitism of the bone hematopoietic stem cell niche. Crit Rev Eukaryot Gene Expr 22(2):131-148

5. Araujo A et al (2014) An integrated computational model of the bone microenvironment in bone-metastatic prostate cancer. Cancer Res 74(9):2391-2401

6. Geiser $\mathrm{T}$ et al (1993) The interleukin-8-related chemotactic cytokines GRO alpha, GRO beta, and GRO gamma activate human neutrophil and basophil leukocytes. J Biol Chem 268(21):15419-15424

7. von Vietinghoff S, Ley K (2008) Homeostatic regulation of blood neutrophil counts. J Immunol 181(8):5183-5188

8. Chervenick PA et al (1968) Quantitative studies of blood and bone marrow neutrophils in normal mice. Am J Physiol 215(2):353-360

9. Lieber JG et al (2004) The in vitro production and characterization of neutrophils from embryonic stem cells. Blood 103(3):852-859

10. Rosales $C$ et al (2017) Neutrophils: their role in innate and adaptive immunity 2017. J Immunol Res 2017:9748345

11. Coffelt SB et al (2016) Neutrophils in cancer: neutral no more. Nat Rev Cancer 16(7):431-446

12. Fridlender ZG et al (2009) Polarization of tumor-associated neutrophil phenotype by TGF-beta: "N1" versus "N2" TAN. Cancer Cell 16(3):183-194

13. Coffelt SB et al (2015) IL-17-producing gammadelta T cells and neutrophils conspire to promote breast cancer metastasis. Nature 522(7556):345-348

14. Heim CE et al (2014) Myeloid-derived suppressor cells contribute to Staphylococcus aureus orthopedic biofilm infection. J Immunol 192(8):3778-3792
15. Cook LM et al (2019) Betaglycan drives the mesenchymal stromal cell osteogenic program and prostate cancer-induced osteogenesis. Oncogene 38(44):6959-6969

16. Casbon AJ et al (2015) Invasive breast cancer reprograms early myeloid differentiation in the bone marrow to generate immunosuppressive neutrophils. Proc Natl Acad Sci USA 112(6):E566-E575

17. Chen Y, Junger WG (2012) Measurement of oxidative burst in neutrophils. Methods Mol Biol 844:115-124

18. Papayannopoulos V (2018) Neutrophil extracellular traps in immunity and disease. Nat Rev Immunol 18(2):134-147

19. Albrengues $\mathbf{J}$ et al (2018) Neutrophil extracellular traps produced during inflammation awaken dormant cancer cells in mice. Science 361(6409): eaao4227

20. Rayes RF et al (2019) Primary tumors induce neutrophil extracellular traps with targetable metastasis promoting effects. JCI Insight 4(16):e128008

21. Wu L et al (2019) Tumor-associated neutrophils in cancer: going pro. Cancers (Basel) 11(4):564

22. Daley JM et al (2008) Use of Ly6G-specific monoclonal antibody to deplete neutrophils in mice. J Leukoc Biol 83(1):64-70

23. Shiozawa $Y$ et al (2011) Human prostate cancer metastases target the hematopoietic stem cell niche to establish footholds in mouse bone marrow. J Clin Invest 121(4):1298-1312

24. Cunningham $D$, You $Z$ (2015) In vitro and in vivo model systems used in prostate cancer research. J Biol Methods 2(1):e17

25. Ahonen TJ et al (2003) Inhibition of transcription factor Stat5 induces cell death of human prostate cancer cells. J Biol Chem 278(29):27287-27292

26. Kwon ED et al (2014) Ipilimumab versus placebo after radiotherapy in patients with metastatic castration-resistant prostate cancer that had progressed after docetaxel chemotherapy (CA184-043): a multicentre, randomised, double-blind, phase 3 trial. Lancet Oncol 15(7):700-712

27. Graff JN et al (2014) Sustained complete response to CTLA-4 blockade in a patient with metastatic, castration-resistant prostate cancer. Cancer Immunol Res 2(5):399-403

28. Gu L et al (2010) Stat5 promotes metastatic behavior of human prostate cancer cells in vitro and in vivo. Endocr Relat Cancer 17(2):481-493

29. Maranto C et al (2018) STAT5A/B blockade sensitizes prostate cancer to radiation through inhibition of RAD51 and DNA repair. Clin Cancer Res 24(8):1917-1931

30. $\mathrm{Li} \mathrm{H}$ et al (2004) Activation of signal transducer and activator of transcription 5 in human prostate cancer is associated with high histological grade. Cancer Res 64(14):4774-4782

31. Fievez L et al (2007) STAT5 is an ambivalent regulator of neutrophil homeostasis. PLoS ONE 2(8):e727

32. Tripathi P et al (2010) STAT5 is critical to maintain effector CD8 + T cell responses. J Immunol 185(4):2116-2124

33. Haddad BR et al (2013) STAT5A/B gene locus undergoes amplification during human prostate cancer progression. Am J Pathol 182(6):2264-2275

34. Janiszewska M et al (2019) Subclonal cooperation drives metastasis by modulating local and systemic immune microenvironments. Nat Cell Biol 21(7):879-888

35. Keeley $\mathrm{T}$ et al (2019) Unmasking the many faces of tumor-associated neutrophils and macrophages: considerations for targeting innate immune cells in cancer. Trends Cancer 5(12):789-798

36. Mackey JBG et al (2019) Neutrophil maturity in cancer. Front Immunol 10:1912

37. Fridlender ZG et al (2012) Transcriptomic analysis comparing tumor-associated neutrophils with granulocytic myeloidderived suppressor cells and normal neutrophils. PLoS ONE $7(2): \mathrm{e} 31524$ 
38. Aarts CEM et al (2019) Neutrophils as suppressors of T Cell proliferation: does age matter? Front Immunol 10:2144

39. Bronte $\mathrm{V}$ et al (2016) Recommendations for myeloid-derived suppressor cell nomenclature and characterization standards. Nat Commun 7:12150

40. Sonpavde G et al (2014) Prognostic impact of the neutrophilto-lymphocyte ratio in men with metastatic castration-resistant prostate cancer. Clin Genitourin Cancer 12(5):317-324

41. Park SI et al (2012) Cyclophosphamide creates a receptive microenvironment for prostate cancer skeletal metastasis. Cancer Res 72(10):2522-2532

42. Lai JJ et al (2012) Androgen receptor influences on body defense system via modulation of innate and adaptive immune systems: lessons from conditional AR knockout mice. Am J Pathol 181(5):1504-1512
43. Salem ML et al (2016) Effect of administration timing of postchemotherapy granulocyte colony-stimulating factor on hostimmune cell recovery and CD8(+) T-cell response. J Immunotoxicol 13(6):784-792

44. Shaul ME, Fridlender ZG (2019) Tumour-associated neutrophils in patients with cancer. Nat Rev Clin Oncol 16(10):601-620

45. Roudier MP et al (2003) Phenotypic heterogeneity of end-stage prostate carcinoma metastatic to bone. Hum Pathol 34(7):646-653

Publisher's Note Springer Nature remains neutral with regard to jurisdictional claims in published maps and institutional affiliations. 